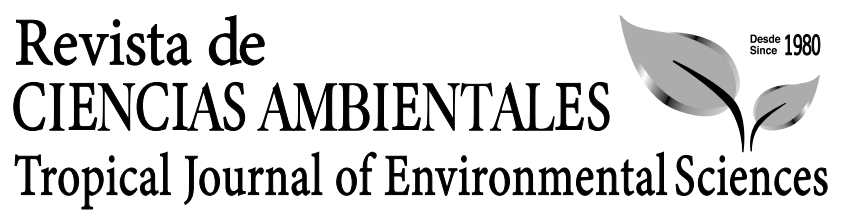

Revista de Ciencias Ambientales (Trop J Environ Sci)

e-ISSN: 2215-3896

(Enero-Junio, 2021) . Vol 55(1): 118-142

DOI: https://doi.org/10.15359/rca.55-1.6

Open Access: www.revistas.una.ac.cr/ambientales e-mail: revista.ambientales@una.ac.cr Rodríguez-Cruz G.

\title{
Escasez de agua en Tegucigalpa, Honduras: ¿Está siendo afectada la subcuenca Guacerique por la variabilidad climática y el arrastre de sedimentos?
}

\author{
Water scarcity in Tegucigalpa, Honduras: Is the Guacerique Subbasin being \\ affected by climate variability and the drag of sediments?
}

\section{German Onil Rodríguez Cruz}

[Recibido: 19 de febrero 2020, Aceptado: 26 de agosto 2020, Corregido: 12 de setiembre 2020, Publicado: 1 de enero 2021]

\section{Resumen}

[Introducción]: En la parte baja de la subcuenca del río Guacerique $\left(210 \mathrm{~km}^{2}\right)$, el embalse Los Laureles (10 millones de metros cúbicos (MMC)), es la fuente de abastecimiento del $30 \%$ de la población de Tegucigalpa, Honduras. El agua del embalse presenta problemas en época lluviosa ligados al incremento de turbidez y color, mientras en época seca, su volumen de agua baja hasta mezclarse con los sedimentos, lo que dificulta su tratamiento. [Objetivo]: El objetivo del estudio fue evidenciar la afectación de la variabilidad climática y el arrastre de sedimentos por medio de la red hídrica de la subcuenca Guacerique, que estima la disponibilidad histórica comparado con la presencia del fenómeno de El Niño-Oscilación del Sur (ENOS) y la relación del arrastre de sedimentos hacia el embalse. [Metodología]: Se realizaron balances hídricos históricos (2001 al 2016) de la subcuenca Guacerique y se calculó la carga de sedimentos. [Resultados]: Se obtuvo una producción histórica de agua de $99.4 \mathrm{MMC}$ de la subcuenca Guacerique. En presencia del fenómeno ENOS la producción fue de 73.4 MMC, en año mezcla Niño/neutral fue 103.8 MMC, en años neutrales fue de 84.3 MMC, en año mezcla Niña/neutral fue de 112.5 MMC y en año mezcla Niño/Niña fue de 216.5 MMC. El aporte de sedimentos al embalse a través de la red hídrica fue de $488.8 \mathrm{~kg} \mathrm{mes}^{-1}$. Se tiene que el $1.7 \%$ del total de sedimentos proviene del río Mateo, el $2.5 \%$ de la quebrada El Ocotal y el $7.5 \%$ de la quebrada el Achiote. [Conclusiones]: Se pudo comprobar mediante los balances hídricos, que la variabilidad climática está afectando los volúmenes de agua en la subcuenca Guacerique, y que junto al arrastre de sedimentos por el río Guacerique, se está reduciendo la capacidad de almacenamiento, lo que provoca menor disponibilidad y escasez de agua en el embalse Los Laureles.

Palabras claves: Balance hídrico; cambio climático; Centroamérica; sedimentación.

\begin{abstract}
[Introduction]: Located in the lower section of the subbasin of the Guacerique River $\left(210 \mathrm{~km}^{2}\right)$, the Los Laureles reservoir (10 million cubic meters (MCM)) supplies $30 \%$ of the population in Tegucigalpa, Honduras. The reservoir faces some problems during the rainy season due to increased turbidity and color, while during the dry season the volume lowers and the water mixes with sediments, hindering treatment. [Objective]: The objective of this study was to evidence the affectation of the climate variability and the drag of sediments to the water network of

1 Graduado de la maestría en Ciencias del Agua del Centro de Investigación para los Recursos Acuáticos (CIRA), Universidad Nacional Autónoma de Nicaragua (UNAN), Managua, onilhn@gmail.com, https://orcid.org/0000-0002-6598-4923
\end{abstract}

\begin{tabular}{|c|c|c|}
\hline 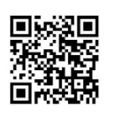 & 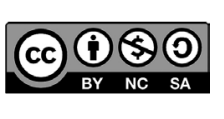 & 118 \\
\hline
\end{tabular}




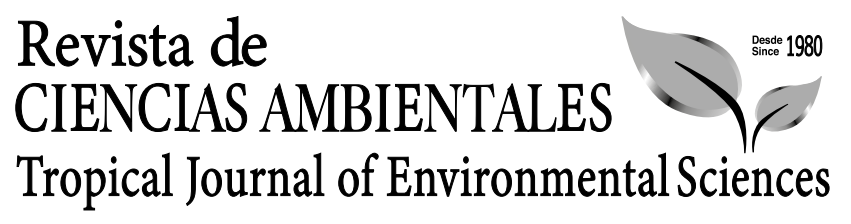

Revista de Ciencias Ambientales (Trop J Environ Sci) e-ISSN: 2215-3896

(Enero-Junio, 2021) . Vol 55(1): 118-142 DOI: https://doi.org/10.15359/rca.55-1.6

Open Access: www.revistas.una.ac.cr/ambientales e-mail: revista.ambientales@una.ac.cr Rodríguez-Cruz G.

the Guacerique subbasin, estimating the historical availability of water compared to the presence of the El Niño Southern Oscillation (ENSO) phenomenon and sediment drag. [Methodology]: Historical water balances (2001 to 2016) of the Guacerique subbasin were obtained, and the sediment load was calculated. [Results]: Historical water production of the Guacerique subbasin was 99.4 MCM. Water production was 73.4 MCM during ENSO, 103.8 MCM during the Niño/Neutral mixed year, 84.3 MCM during neutral years, 112.5 MCM during the Niña/Neutral mixed year, and 216.5 MCM during the Niño/Niña mixed year. The sediment contribution to the dam through the hydric network was $488.8 \mathrm{~kg} \mathrm{month}^{-1}$. A total of $1.7 \%$ of sediments came from the Mateo River, $2.5 \%$ from the El Ocotal brook, and $7.5 \%$ from the El Achiote brook. [Conclusions]: Using hydric balances, climate variability is confirmed to affect water volumes in the Guacerique subbasin. Due to the dragging of sediments in the Guacerique River, storage capacity is reduced, resulting in less water availability and scarcity in the reservoir.

Keywords: Central America; climate change; hydric balance; sedimentation.

\section{Introducción}

Los componentes del ciclo hidrológico presentan una variabilidad natural notable en escalas de tiempo interanuales a decadales que enmascara frecuentemente las tendencias a largo plazo (Bates et al., 2008). Los recursos hídricos, tanto en términos de cantidad como de calidad, están gravemente afectados por las actividades humanas, en particular por la agricultura, cambios de uso del suelo, construcción y gestión de embalses, emisiones de sustancias contaminantes y tratamiento de aguas de desecho (Bates et al., 2008). Además de las actividades humanas, las condiciones naturales como la geomorfología y los aumentos en los regímenes de precipitación contribuirán con el incremento de partículas sólidas en suspensión (turbidez) en lagos y embalses, debido a la erosión hídrica del suelo (Leemans y Kleidon, 2002) e incorporarán sustancias contaminantes (Bouraoui et al., 2004; Neff et al., 2000; Mimikou et al., 2000).

Honduras cuenta con ocho embalses mayores de $100000 \mathrm{~m}^{3}$ (PREPAC, 2005) y dos de ellos están ubicados en la ciudad capital, los cuales abastecen el 80 \% de agua potable a la población de Tegucigalpa (SANAA, 2004). El embalse Los Laureles, se encuentra dentro de la subcuenca Guacerique, en el departamento de Francisco Morazán, donde se desarrolló la investigación, fue construido en 1976 y abastece de agua potable al $30 \%$ de la población de Tegucigalpa (350 000 personas aproximadamente) y es administrado por el Servicio Autónomo Nacional de Acueductos y Alcantarillados (SANAA), con un volumen máximo de 10 MMC.

La subcuenca Guacerique fue declarada como "Zona Forestal Protegida” según el acuerdo No. 03-73 del 02 de enero de 1973 (Depto. de Cuencas Hidrográficas y Ambiente del ICF, 2012-2017). De acuerdo con las zonas de vida de Holdridge y la hidrología del embalse, con los datos suministrados por la estación del Batallón cercano al embalse, su altitud es $1050 \mathrm{msnm}$, bio-temperatura media: $22.5^{\circ} \mathrm{C}$, precipitación total promedio anual: $966 \mathrm{~mm}$, zona de vida: bs-PSt (Bosque seco pre montano subtropical), evapotranspiración potencial (ETP): $1332 \mathrm{~mm}$, relación evapotranspiración potencial (rETP): 1.38 y provincia de humedad: subhúmedo.

\begin{tabular}{|c|c|c|}
\hline 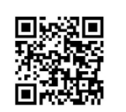 & (c) (1) (9) & 119 \\
\hline
\end{tabular}




\section{Revista de CIENCIAS AMBIENTALES Tropical Journal of Environmental Sciences}

Revista de Ciencias Ambientales (Trop J Environ Sci) e-ISSN: 2215-3896

(Enero-Junio, 2021) . Vol 55(1): 118-142 DOI: https://doi.org/10.15359/rca.55-1.6

Open Access: www.revistas.una.ac.cr/ambientales e-mail: revista.ambientales@una.ac.cr Rodríguez-Cruz G.

Se sabe que "El Niño y La Niña" son las fases cálidas y frías de un patrón climático recurrente a través del Pacífico tropical, el cual se le conoce como ENOS (NOAA, 2016). El fenómeno ENOS ha afectado Centroamérica con sequías y estaciones lluviosas irregulares, y con repercusiones económicas y sociales. Además, la deforestación se considera otro problema importante para los recursos hídricos, ya que la preservación de los bosques conserva estos recursos y evita inundaciones. De acuerdo con el Panel Intergubernamental del Cambio Climático (IPCC, 2008), una de las predicciones obtenidas para Honduras es que experimentará estrés hídrico severo en áreas de las regiones intermontanas septentrional, central y occidental del país. En esas áreas, el abastecimiento de agua y la generación de energía hidroeléctrica podrían resultar gravemente afectados por el cambio climático (Bates et al., 2008).

Las ciudades de Tegucigalpa y Comayagüela forman una conurbación conocida como el Distrito Central y cuenta aproximadamente con 1.4 millones de habitantes (Proyección INE, 2015). Los habitantes de esta zona utilizan los recursos hídricos superficiales como la principal fuente de abastecimiento. Sin embargo, estos son los más vulnerables al deterioro de su calidad y disponibilidad, hecho que se ha vuelto una realidad en los años recientes. Durante el periodo lluvioso, el embalse Los Laureles presenta problemas ligados al incremento de la turbidez y color, por ende, dificultades en el tratamiento, pues se sobrepasa la capacidad de dosificación de las plantas de tratamiento de cloro y floculantes. En época seca, el nivel del embalse desciende tan drásticamente (entre 15 y $20 \mathrm{~m}$ ) que el agua disponible casi se mezcla con el sedimento depositado en el fondo, con lo cual provoca problemas de mal olor, y alto contenido de hierro y manganeso.

En el pasado, los embalses del país han sido expuestos a eventos extremos meteorológicos (Huracán Mitch en 1998 y depresiones tropicales en el 2010) y esto ha acentuado el problema de deforestación que produce arrastre de sedimento, lo cual provoca aumento en la turbidez y el color en los ríos y afluentes que los abastecen. La deforestación en la subcuenca del río Guacerique ha incrementado la sedimentación en el embalse, se ha reducido la capacidad de abastecimiento y se ha aumentado la contaminación del agua, debido a las urbanizaciones construidas (Berrios, 2014). En muchos países, la distribución del agua está condicionada según la región geográfica, y Honduras no es la excepción. La costa atlántica es la que posee la mayor cantidad de agua per cápita del país; el corredor seco, conformado por las regiones central y sur, se conoce como el que tiene menos y de esta área la subcuenca del río Guacerique forma parte y sus acuíferos son de baja productividad.

Tegucigalpa se abastece de tres fuentes superficiales principales con una cantidad total de 55 MMC: La represa La Concepción en el suroeste de la capital (36 MMC de almacenamiento, lo que representa un $50 \%$ del abastecimiento), la represa Los Laureles en el río Guacerique, al oeste de la zona metropolitana de Tegucigalpa (10 MMC de almacenamiento, lo que representa un $30 \%$ del abastecimiento) y las fuentes en El Picacho, en el parque nacional La Tigra al noreste de la capital con un $20 \%$ del abastecimiento.

En un estudio de 1998 del SANAA, se estimó que la demanda para abastecer Tegucigalpa en el año 2010 sería aproximadamente entre 78 y 102 MMC anuales. Según el SANAA, se requiere

\begin{tabular}{|c|c|c|}
\hline 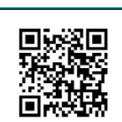 & (c) (1) () (2) & 120 \\
\hline
\end{tabular}




\section{Revista de CIENCIAS AMBIENTALES Tropical Journal of Environmental Sciences}

Revista de Ciencias Ambientales (Trop J Environ Sci) e-ISSN: 2215-3896

(Enero-Junio, 2021) . Vol 55(1): 118-142 DOI: https://doi.org/10.15359/rca.55-1.6

Open Access: www.revistas.una.ac.cr/ambientales e-mail: revista.ambientales@una.ac.cr Rodríguez-Cruz G.

la construcción de dos represas con una capacidad de 100 MMC cada una para abastecer la capital de manera permanente. El déficit de agua en la ciudad se estima en $50 \%$ y obedece a la poca capacidad de los actuales embalses, así como a las fugas del sistema de distribución. También es conocido que Tegucigalpa se abastece en un $100 \%$ de aguas superficiales durante la época lluviosa y en un $95 \%$ en la época seca, lo cual aumenta su vulnerabilidad. El restante $5 \%$, durante la época seca, viene de pozos propiedad del SANAA y de propiedad privada. Con base en lo anterior, el objetivo de este estudio fue estimar la disponibilidad histórica (2001 al 2016) del agua a través de balances hídricos del embalse Los Laureles y el transporte de sedimentos a través de los tributarios por medio de la colocación de trampas de sedimentos.

\section{Metodología}

\section{1 Área de estudio}

El área comprendió la parte baja de la subcuenca del río Guacerique $\left(210 \mathrm{~km}^{2}\right)$, la cual forma parte de la cuenca del río Choluteca que drena hacia el océano Pacífico (Figura 1). Se ubica entre las coordenadas $14^{\circ} 00^{\prime} 30^{\prime \prime}$ y $14^{\circ} 14^{\prime} 00^{\prime \prime}$ latitud norte y $87^{\circ} 23^{\prime} 49^{\prime \prime}$ y $87^{\circ} 40^{\prime} 00^{\prime \prime}$ longitud oeste. El río principal de la subcuenca de nombre homónimo (Guacerique) nace en las montañas de Yerbabuena con una producción aproximada de $84 \mathrm{MMC} /$ año, que representa el $37 \%$ del total de la producción de agua de la cuenca alta del río Choluteca (SANAA, 2011).

Dentro del área de estudio se localiza el espejo de agua del embalse Los Laureles $\left(1 \mathrm{~km}^{2}\right)$, entre las coordenadas $14^{\circ} 03^{\prime} 40^{\prime \prime}$ y $14^{\circ} 06^{\prime} 40^{\prime \prime} \mathrm{N}$; y $87^{\circ} 14^{\prime} 51^{\prime \prime}$ y $87^{\circ} 14^{\prime} 23^{\prime \prime} \mathrm{O}$ (Figura 1) y una altura de $1041 \mathrm{msnm}$. Este embalse cuenta con una capacidad de producción promedio de $450 \mathrm{ls}^{-1}$ y un tanque de almacenamiento de $7570 \mathrm{~m}^{3}$.

\begin{tabular}{|c|c|c|}
\hline 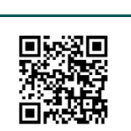 & (c) (i) (3) () () & 121 \\
\hline
\end{tabular}



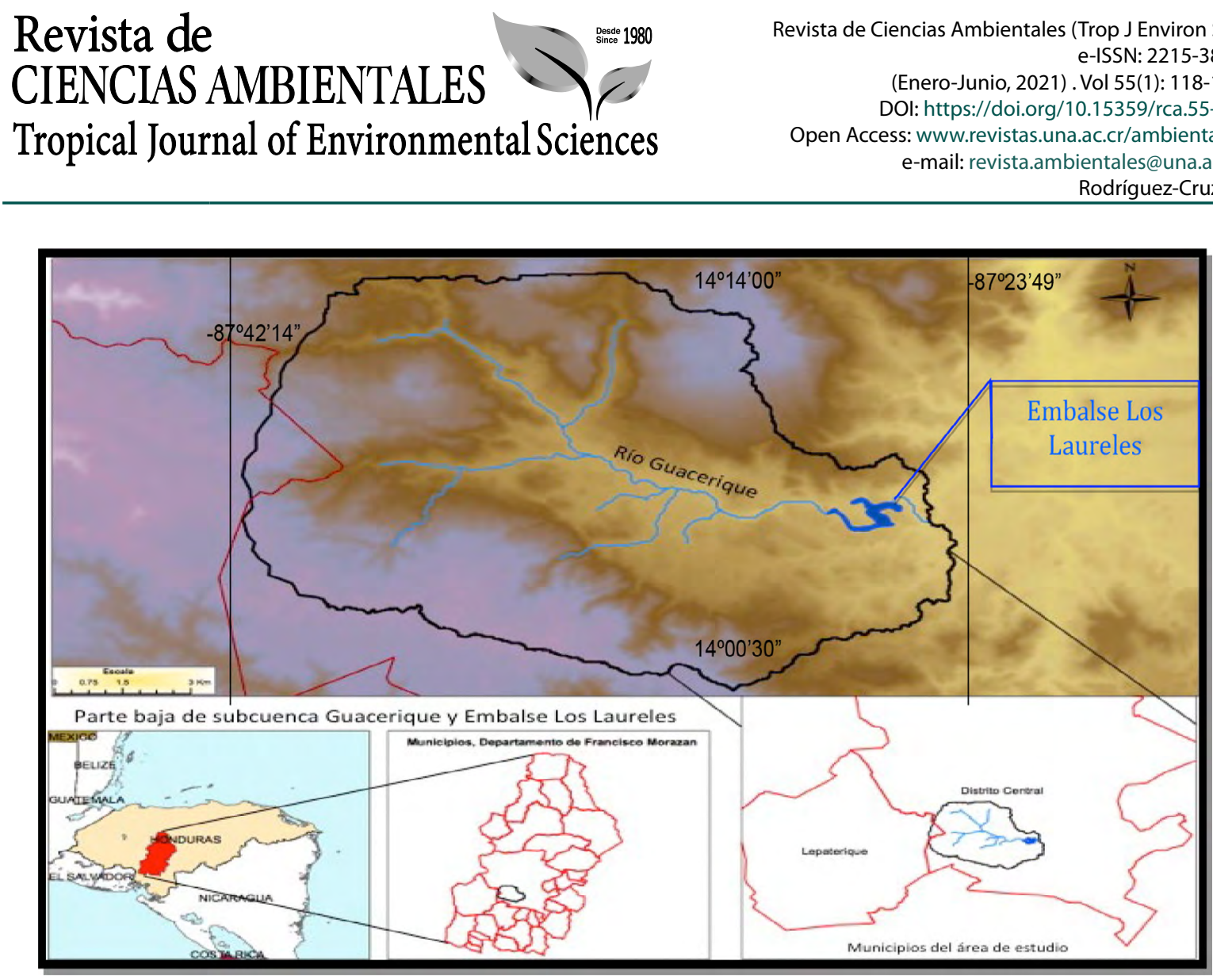

Figura 1. Mapa de la subcuenca Guacerique y embalse Los Laureles, Tegucigalpa, Honduras.

Figure 1. Map of Guacerique sub-basin and the Los Laureles reservoir, Tegucigalpa, Honduras.

\subsection{Balance hídrico}

Las técnicas del balance hídrico son uno de los principales objetivos en hidrología, son un medio para solucionar importantes problemas hidrológicos teóricos y prácticos (Sokolov y Chapman, 1981). Los balances hídricos son imprescindibles en los estudios de regulación de cuencas, embalses y en los proyectos de suministros de agua para acueducto, riego y generación hidroeléctrica (Fattorelli y Fernández, 2011). Las principales variables en el balance hídrico son: precipitación, evapotranspiración, escurrimiento superficial (escorrentía), almacenamiento superficial y subterráneo y flujo de agua subterránea. La precipitación se considera como la principal variable de entradas al sistema hídrico. La evapotranspiración es el fenómeno de cambio de estado físico de líquido a gaseoso, incluye la evaporación de la cobertura vegetal.

La escorrentía es el agua de las precipitaciones que no es evaporada ni infiltrada, escurre superficialmente en forma de escorrentía directa y basal, y es afectada por consecuencias climáticas como la intensidad y la duración de la precipitación. La intensidad resulta fundamental porque se relaciona con la tasa de infiltración del suelo. Si la intensidad es mayor a la infiltración, todo el excedente de la lluvia es escorrentía superficial. Uno de los métodos más utilizado en la elaboración de un balance hídrico es el de aproximación, por el método de "Thornthwaite

\begin{tabular}{|c|c|c|}
\hline 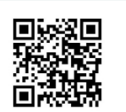 & (c) (i) (9) & 122 \\
\hline
\end{tabular}




\section{Revista de CIENCIAS AMBIENTALES Tropical Journal of Environmental Sciences}

Revista de Ciencias Ambientales (Trop J Environ Sci) e-ISSN: 2215-3896 (Enero-Junio, 2021) . Vol 55(1): 118-142 DOI: https://doi.org/10.15359/rca.55-1.6 Open Access: www.revistas.una.ac.cr/ambientales e-mail: revista.ambientales@una.ac.cr Rodríguez-Cruz G.

(1948)”. A continuación se resumen los componentes que utiliza este método. El cálculo del índice de calor mensual (i) con las temperaturas medias diarias del aire, con la Ecuación 1.

$$
i=(t / 5) \wedge 1.514(\mathbf{E} . \mathbf{1})
$$

obteniendo el índice de calor anual (I) como la suma de los doce valores del índice de calor mensual para meses teóricos de 30 días y 12 horas diarias de sol. A partir de estos datos propone la siguiente Ecuación 2.

$$
E T P=1.6(10 t / I) a(\text { E. 2) }
$$

$\mathrm{ETP}=$ Evapotranspiración potencial mensual no corregida en $\mathrm{mm} /$ día

$\mathrm{t}=$ Temperatura media mensual en ${ }^{\circ} \mathrm{C}$

I= Índice de calor anual

a= Ecuación 3 de I igual a

$I=675 * 10-9 I 3-771 * 10-7 I 2+1.972 * 10-5 I+0,49239$ (E. 3)

La evapotranspiración real (ETR) se calcula a partir de tres factores, dos ya conocidos, la ETP, la precipitación e infiltración efectiva (ie). La evapotranspiración real (ETR) es el volumen de agua que realmente se evapotranspira en el mes, depende del agua disponible para evaporar. Una vez calculada la ETR podemos conocer la precipitación útil (PU) mediante la Ecuación 4:

$$
P U=P-E T R(\text { E.4) }
$$

La (PU) tiene dos componentes, escorrentía (E) superficial e infiltración (IF). El volumen infiltrado se calcula para períodos de larga duración (un mes o un año). Custodio y Llamas (1983) citan una Ecuación 5 empírica aplicada con éxito en regiones semiáridas, del tipo:

$$
I F=\propto(P-P 0)(\text { E. 5) }
$$

IF = Infiltración en mm año ${ }^{-1}$

$\propto=$ Coeficiente adimensional característico de la cuenca

$\mathrm{P}=$ Precipitación sobre la cuenca en $\mathrm{mm}$ año ${ }^{-1}$

$\mathrm{Po}=$ Valor en $\mathrm{mm} / \mathrm{año}$ característico de la cuenca

El método de Thornthwaite parte, para su cálculo, de los siguientes supuestos:

1. El período considerado es el año hidrológico, de octubre a septiembre.

2. La temperatura y la precipitación se aplican como valores medios mensuales.

3. La infiltración eficaz del suelo, al comienzo del año hidrológico, es 0 y las variaciones de dicha reserva se deben exclusivamente al resultado del balance, Ecuación 6:

\begin{tabular}{|c|c|c|}
\hline 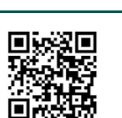 & (c) (1) () (2) (2) & $\sqrt{123}$ \\
\hline
\end{tabular}

$$
P-E T R= \pm R U \text { (E. 6) }
$$

El balance hídrico se llevó a cabo mediante la aplicación del método de Thornthwaite (1948), para lo cual se tomó en cuenta la información suministrada de cinco estaciones meteorológicas 


\section{Revista de CIENCIAS AMBIENTALES Tropical Journal of Environmental Sciences}

Revista de Ciencias Ambientales (Trop J Environ Sci) e-ISSN: 2215-3896

(Enero-Junio, 2021) . Vol 55(1): 118-142 DOI: https://doi.org/10.15359/rca.55-1.6

Open Access: www.revistas.una.ac.cr/ambientales e-mail: revista.ambientales@una.ac.cr Rodríguez-Cruz G.

del SANAA para los años 2001 al 2016. Un año hidrológico comprende desde el mes de octubre hasta el mes de septiembre del siguiente año. La Ecuación 7 básica del método Thornthwaite (1948) queda de la siguiente manera:

$$
B H=P-(E+E T R+Q)+\eta(\mathbf{E . ~ 7 )}
$$

La estación de Rincón de Dolores se instaló a mediados del año 2004, por lo que no se contó con información para los tres primeros años hidrológicos. Mientras que la estación El Escarbadero se instaló también a mediados de 2004 y dejó de funcionar en diciembre de 2012. Por tanto, los años hidrológicos del período 2001 al 2004 se hicieron solo con tres (3) estaciones y el período de 2013 al 2016, se hizo con cuatro (4) estaciones. No se llevó a cabo relleno de las series de datos climáticos, pues se contaba con toda la información completa. Debe tenerse en cuenta que en la definición exacta de los componentes del balance hídrico implica la introducción en la ecuación de un término residual de discrepancia $(\eta)$, encargado de recoger los errores sistemáticos y la influencia de factores desconocidos o que no se tuvieron en cuenta y que pueden afectarlo significativamente. El valor de discrepancia $\eta$ se consideró aceptable si era menor al $10 \%$, e ideal si era menor al $7 \%$. Se realizó un balance hídrico por año y uno consolidado para los 16 años.

\subsection{Usos del agua}

Desde su creación en 1972, el uso principal del agua del embalse Los Laureles es la de abastecimiento potable para la población del suroeste de Tegucigalpa. Sin embargo, se considera ampliar el uso de protección de la vida acuática y recreación para su aprovechamiento turístico en un futuro, de acuerdo con las autoridades del SANAA en el 2016. Las características morfológicas y morfométricas se detallan en los Cuadros 1 y 2.

Cuadro 1. Características morfológicas de la subcuenca Guacerique.

Table 1. Morphological characteristics of the Guacerique sub-basin.

\begin{tabular}{ll}
\hline \multicolumn{1}{c}{ Característica } & \multicolumn{1}{c}{ Cantidad } \\
\hline Superficie de la subcuenca $\left(\mathrm{km}^{2}\right)$ & 210.1 \\
Perímetro de la subcuenca $(\mathrm{km})$ & 66.7 \\
Cota máxima (msnm) & 2000 \\
Cota mínima (msnm) & 1020 \\
Altura media (msnm) & 1426 \\
Pendiente media (\%) & 17 \\
Máximo orden del río & 4 \\
Longitud del río principal (km) & 27 \\
Tiempo de concentración (horas) & 4.1 \\
\hline
\end{tabular}

\begin{tabular}{|c|c|c|}
\hline 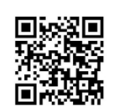 & (c) (1) (9) & 124 \\
\hline
\end{tabular}




\section{Revista de CIENCIAS AMBIENTALES Tropical Journal of Environmental Sciences}

Revista de Ciencias Ambientales (Trop J Environ Sci) e-ISSN: 2215-3896

(Enero-Junio, 2021) . Vol 55(1): 118-142 DOI: https://doi.org/10.15359/rca.55-1.6

Open Access: www.revistas.una.ac.cr/ambientales e-mail: revista.ambientales@una.ac.cr Rodríguez-Cruz G.

Cuadro 2. Características morfométricas del embalse Los Laureles.

Table 2. Morphometric characteristics of the Los Laureles reservoir.

\begin{tabular}{lll}
\hline \multicolumn{1}{c}{ Característica } & \multicolumn{1}{c}{ Cantidad } \\
\hline Superficie del embalse (estiaje, $\mathrm{km}^{2}$ ) & 0.54 & \\
Superficie del embalse (época lluviosa, $\left.\mathrm{km}^{2}\right)$ & 1 & \\
Capacidad total $(\mathrm{MMC})$ & 10 & \\
Profundidad máxima $(\mathrm{m})$ & 37 & \\
\hline
\end{tabular}

\subsection{Selección de los años Niño, Niña, Neutral y Mezcla}

Este estudio (2001 a 2016) se hizo con base en la clasificación de los resúmenes mensuales de la situación de ENOS (El Niño Oscilación Sur o Austral, por sus siglas en inglés) según el índice NINO 3.4 (120- 170W, 5S-5N) del Instituto Internacional de Investigación para el Clima y la Sociedad (IRI, por sus siglas en inglés) de la Universidad de Columbia, de los Estados Unidos (http://iri.columbia.edu/our-expertise/climate/forecasts/enso/current/).

Debido a que los eventos ENOS suelen tener diferentes intensidades, cobertura y estacionalidad, no hay un acuerdo unánime sobre lo que constituye el fenómeno; sin embargo, existen amplio consenso entre grupos investigadores de diferentes instituciones. Cada fase del Niño o la Niña provoca perturbaciones predecibles de temperatura, precipitación y vientos (NOAA, 2016). Estos fenómenos suelen persistir entre 9 y 12 meses, aunque en ciertas ocasiones han llegado a persistir por hasta dos años.

Basado en el anterior, se usaron las clasificaciones mensuales para realizar una anual del fenómeno ENOS, para luego compararlo con el resultado anual del balance hídrico para la subcuenca Guacerique. Resultaron las siguientes combinaciones: años Niño, años mezcla (Niño-Neutral / Neutral-Niño), años Neutrales, años Niña, años mezcla (Niña-Neutral / Neutral-Niña) y años mezcla (Niño-Niña / Niña-Niño). Para clasificar un año mezcla, se debían tener al menos cinco meses de un fenómeno y el resto de los meses de otro.

\subsection{Trampas de sedimentos}

El transporte y carga de estos materiales se estimó mediante la colocación de siete trampas de sedimentos: dos en el río Guacerique y cinco en sus tributarios, por un tiempo de 21 días durante el mes de junio de 2016. Los sitios seleccionados debieron cumplir los siguientes requisitos: (1) Cauce del río bien definido, evitando la sinuosidad, (2) Lecho del río compuesto por material permeable, (3) Caudal moderado que permitiera colocar la trampa y (4) colocarse a suficiente profundidad para que la trampa quedara bajo agua (López, 1990).

Las trampas estaban hechas de tubos de PVC de 1 metro de largo por 8 pulgadas de ancho y con una entrada y salida de agua de 1 pulgada de ancho (Figura 2). Se midió el caudal del río, también se insertaron dos varillas de hierro en el centro del río con un mazo y a continuación se colocó la trampa en el centro del cauce. Luego se retiró la trampa después de tres semanas para tener una muestra representativa. La muestra colectada se dejó sedimentar durante una semana

\begin{tabular}{|c|c|c|}
\hline 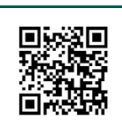 & (c) (i) () (2) & 125 \\
\hline
\end{tabular}




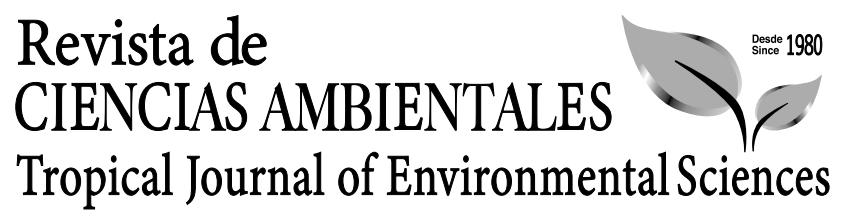

Revista de Ciencias Ambientales (Trop J Environ Sci) e-ISSN: 2215-3896 (Enero-Junio, 2021) . Vol 55(1): 118-142 DOI: https://doi.org/10.15359/rca.55-1.6 Open Access: www.revistas.una.ac.cr/ambientales e-mail: revista.ambientales@una.ac.cr Rodríguez-Cruz G.

en un envase de 10 litros. Posteriormente se agregó el material sedimentado a una cápsula de porcelana grande y antes se anotó el peso de la cápsula de porcelana vacía. Se pesó la cápsula de porcelana con la muestra húmeda y se anotó el peso. Se secó la muestra en el horno a $105^{\circ} \mathrm{C}$ durante 24 horas. Se pesó la muestra seca y se anotó el peso. Los resultados obtenidos se expresaron en gramos $(\mathrm{g})$.

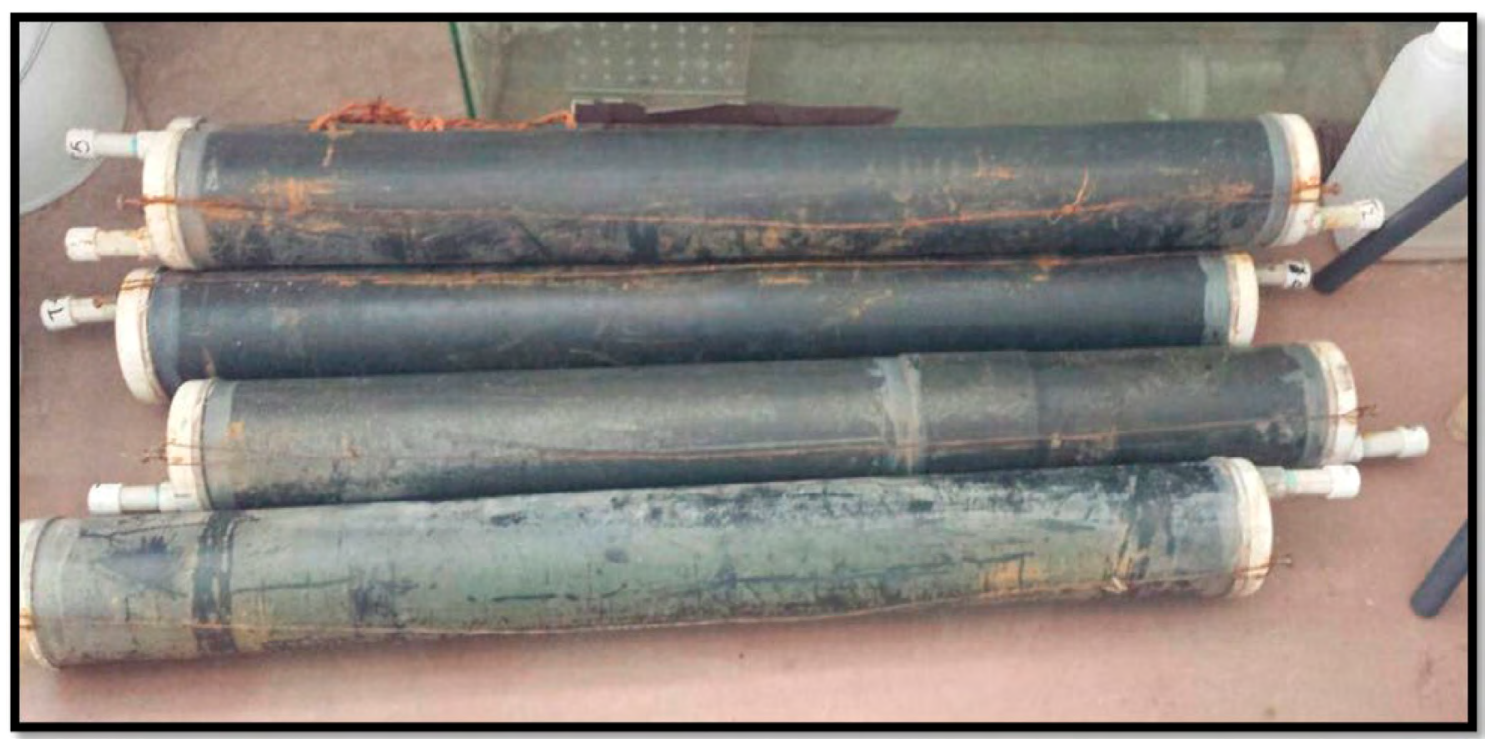

Figura 2. Trampas de sedimentos hechas de tubos PVC.

Figure 2. Sediment traps made of PVC pipes.

\section{Resultados y discusión}

Se presentan los insumos del balance histórico que incluye el periodo de octubre del 2000 a septiembre del 2016. De acuerdo con las bases de datos utilizadas en este estudio, la precipitación promedio anual de la subcuenca es de $1180.5 \mathrm{~mm}$ y la temperatura promedio varía entre $20.2^{\circ} \mathrm{C}$ en las zonas altas y $23.1^{\circ} \mathrm{C}$ en la zona baja conocida como el Batallón, aproximadamente a un kilómetro antes de llegar al embalse Los Laureles y la evapotranspiración promedio es de $644 \mathrm{~mm}$; sin embargo, esta varió en cada estación meteorológica.

\subsection{Precipitación 2001-2016}

La ubicación de las estaciones meteorológicas en la subcuenca Guacerique se muestra a continuación (Figura 3); de las cuales se utilizaron cinco bases de datos disponibles; 1. Batallón, 2. Quiebramontes, 3. El Escarbadero, 4. Rincón de Dolores y 5. San Matías. Para la subcuenca Guacerique se presentaron máximos de precipitación registrados de la siguiente manera, en la estación de "Quiebramontes" con 1222.7 mm, seguido de la estación de "San Matías" con 1112.7 mm ambas

\begin{tabular}{|c|c|c|}
\hline 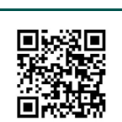 & (c) (1) () () (2) & 126 \\
\hline
\end{tabular}




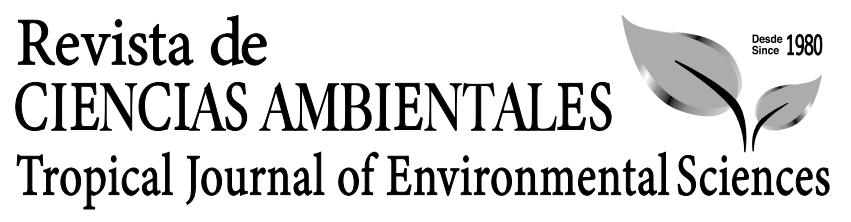

Revista de Ciencias Ambientales (Trop J Environ Sci) e-ISSN: 2215-3896 (Enero-Junio, 2021) . Vol 55(1): 118-142 DOI: https://doi.org/10.15359/rca.55-1.6 Open Access: www.revistas.una.ac.cr/ambientales e-mail: revista.ambientales@una.ac.cr Rodríguez-Cruz G.

estaciones meteorológicas en el año 2014, mientras en la estación de "Batallón" con 1138.9 mm en el 2007 y en las estaciones de "Escarbadero y Rincón de Dolores" fue de $1056.2 \mathrm{~mm}$ en el año 2016. Los mínimos que se registraron fueron de $839 \mathrm{~mm}$ en las estaciones de "Escarbaderos" y "Rincón de Dolores", ambas en el 2004, mientras que en la estación de "San Matías" fue de 872.9 mm en el 2001, en la estación "Quiebramontes" fue de $961.8 \mathrm{~mm}$ igualmente en el año $2001 \mathrm{y}$, por último, en la estación "Batallón” con 1045.5 mm en el 2013 (Apéndices 1 al 5).
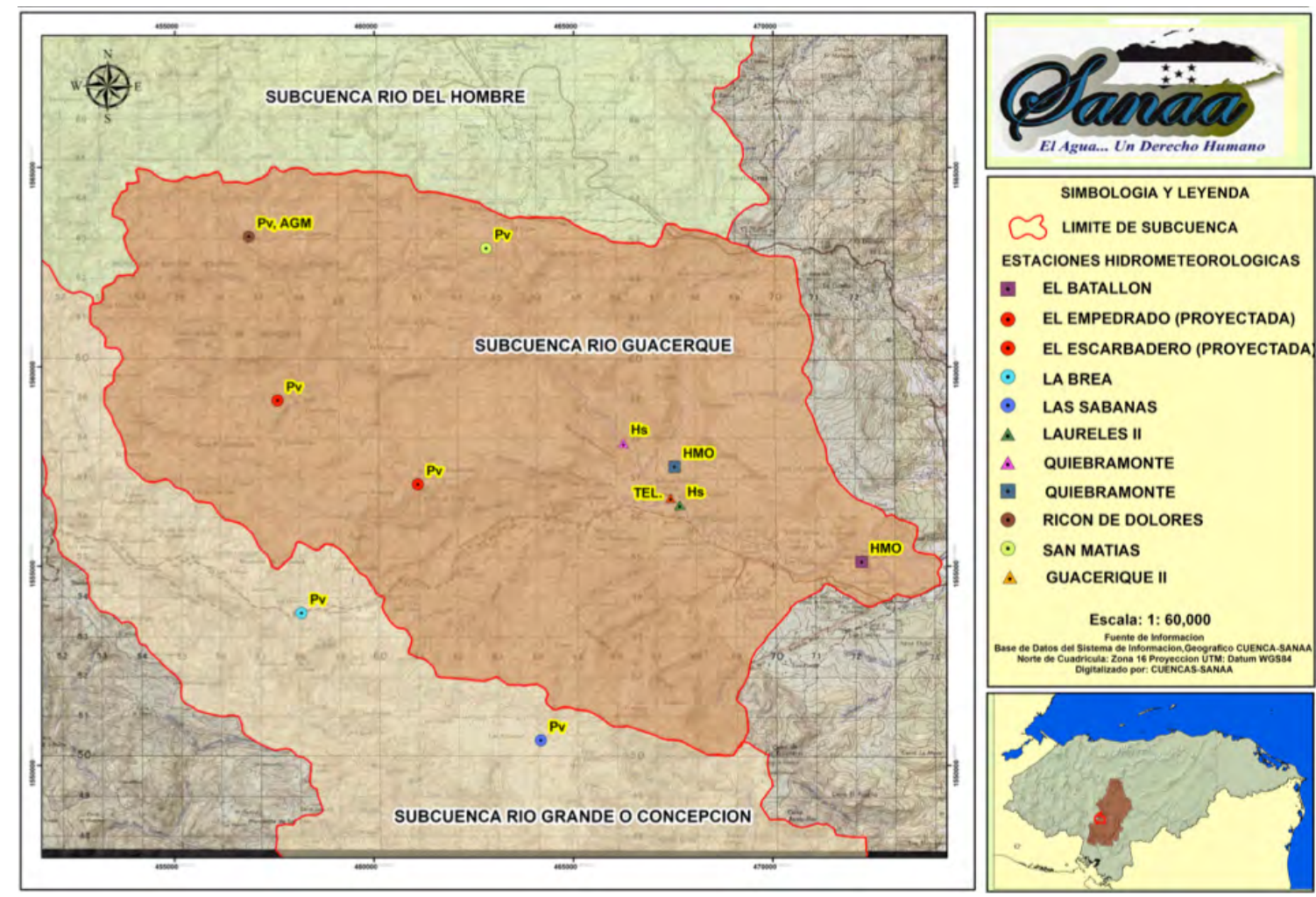

Figura 3. Mapa de la red de estaciones meteorológicas de la subcuenca Guacerique. Fuente: Departamento de Cuencas Hidrográficas, SANAA.

Figure 3. Map of the network of meteorological stations of Guacerique sub-basin. Source: Department of hydrography basins, SANAA.

\subsection{Temperatura 2001-2016}

En la temperatura promedio anual de las cinco (5) estaciones meteorológicas se observó un incremento de la temperatura especialmente en los años 2015 y 2016. Cuando la temperatura ambiente aumenta, provoca una mayor evaporación, debido a mayor disponibilidad de energía calórica. Cuando se tiene mayor energía calórica en el aire como en 2015 y 2016, esta aumenta la

\begin{tabular}{|c|c|c|c|c|c|}
\hline 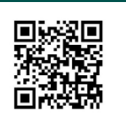 & (c) (i) (5) () & $\overbrace{\text { AMBEETIIIES }}$ & $\frac{O \%}{20}$ & 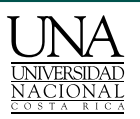 & 127 \\
\hline
\end{tabular}




\section{Revista de CIENCIAS AMBIENTALES Tropical Journal of Environmental Sciences}

Revista de Ciencias Ambientales (Trop J Environ Sci) e-ISSN: 2215-3896

(Enero-Junio, 2021) . Vol 55(1): 118-142 DOI: https://doi.org/10.15359/rca.55-1.6

Open Access: www.revistas.una.ac.cr/ambientales e-mail: revista.ambientales@una.ac.cr Rodríguez-Cruz G.

capacidad del aire para retener humedad. Por ello es de suma importancia contar con este parámetro meteorológico, ya que con este se puede estimar tanto la temperatura media mensual y la como media anual, que forman parte del balance hídrico. Los datos de temperatura proporcionados por el SANAA fueron medias máximas y mínimas mensuales, las cuales se promediaron para obtener la temperatura media mensual y la temperatura media anual, que es el promedio de las medias mensuales para cada año (Apéndices 6 al 10). Nótese la variación de temperatura media mensual entre estaciones, debido a su ubicación de acuerdo con la altitud, las cuales resultaron mayores en Batallón y Quiebramontes y menores en Rincón de Dolores y San Matías.

\subsection{Balance hídrico histórico (2001-2016)}

A continuación, se presenta un balance histórico que incluye el periodo de octubre del 2000 a septiembre del 2016. Para el balance hídrico histórico se utilizó la información histórica de cinco (5) estaciones meteorológicas presentes en la zona (Cuadro 3). El período de registros para este balance comprende los años hidrológicos de octubre del 2000 hasta septiembre 2016.

Con la metodología de Thornthwaite (1948) modificada, se obtuvo un balance hídrico histórico; para ello se tomaron los promedios anuales de temperatura y precipitación. Es decir, se tomó la precipitación del mes octubre de cada año hidrológico (16 años en total) y se obtuvo el promedio y así sucesivamente para cada mes, y se realizó un balance histórico consolidado al obtener los promedios de las cinco estaciones meteorológicas y un promedio de los 16 años para crear un balance hídrico final, el cual dio un nivel de discrepancia de $5.4 \%$ (Cuadro 4).

Cuadro 3. Balance hídrico consolidado de la sub-cuenca Guacerique (2001-2016)

Table 3. Consolidate Hydric balance of the Guacerique sub-basin (2001-2016).

\begin{tabular}{ccccccc}
\hline Periodo & $\begin{array}{c}\text { Precipitación } \\
(\mathbf{m m})\end{array}$ & $\begin{array}{c}\text { ETR } \\
(\mathbf{m m})\end{array}$ & $\begin{array}{c}\text { Excedente } \\
(\mathbf{m m})\end{array}$ & $\begin{array}{c}\text { Excedente } \\
(\mathbf{M M C})\end{array}$ & $\begin{array}{c}\boldsymbol{\eta} \\
(\mathbf{m m})\end{array}$ & $\begin{array}{c}\boldsymbol{\eta} \\
\mathbf{\%}\end{array}$ \\
\hline 2001 al 2016 & 1180.5 & 644.1 & 473.2 & 99.4 & 63.2 & 5.4 \\
\hline
\end{tabular}

$\eta=$ discrepancia, $\mathrm{ETR}=$ Evapotranspiración real, $\mathrm{MMC}=$ Millones de Metros Cúbicos

A continuación, se muestran los balances hídricos anuales consolidados para la subcuenca del río Guacerique, además de una columna adicional informativa sobre el estatus de fenómeno climatológico El Niño Oscilación del Sur (ENOS, por sus siglas en inglés) que se presentó para cada año de estudio y el número de estaciones meteorológicas que se tuvieron disponibles para cada periodo en cuestión (Cuadro 5). La selección de los años Niño, Niña y Neutral para este estudio (2001 a 2016) se hizo con base en la clasificación de los resúmenes mensuales de la situación de ENOS, según el índice NINO3.4 (120-170W, 5S-5N) del IRI de la Universidad de Columbia de los Estados Unidos, por lo que en algunos años hidrológicos se puede observar su transición (mezcla) (http://iri.columbia.edu/our- expertise/climate/forecasts/enso/current/).

Estos fenómenos suelen persistir entre 9 y 12 meses, aunque en ciertas ocasiones han llegado a persistir por hasta dos años. Es importante tener en cuenta que El Niño y La Niña no

\begin{tabular}{|c|c|c|}
\hline 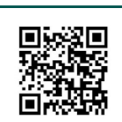 & (c) (i) () (2) & 128 \\
\hline
\end{tabular}




\section{Revista de CIENCIAS AMBIENTALES Tropical Journal of Environmental Sciences}

Revista de Ciencias Ambientales (Trop J Environ Sci) e-ISSN: 2215-3896

(Enero-Junio, 2021) . Vol 55(1): 118-142 DOI: https://doi.org/10.15359/rca.55-1.6 Open Access: www.revistas.una.ac.cr/ambientales e-mail: revista.ambientales@una.ac.cr Rodríguez-Cruz G.

son los únicos factores que condicionan las características climáticas a escala mundial, y que la intensidad del ENOS no se corresponde sistemáticamente con la de los efectos. Respecto a la escala regional, las proyecciones estacionales deberán tener en cuenta los efectos relativos tanto del estado del fenómeno ENOS como de otros condicionantes climáticos pertinentes a escala local (OMM, 2018).

Cuadro 4. Resumen de balance hídrico anual del periodo 2001 al 2016 con estatus de fenómeno climatológico Table 4. Summary of annual hydric balances from 2001 to 2016 with the status of climatological phenomenon.

\begin{tabular}{cccccccc}
\hline $\begin{array}{c}\text { Periodo y \# de } \\
\text { estaciones }\end{array}$ & $\begin{array}{c}\text { Precipitación } \\
(\mathbf{m m})\end{array}$ & $\begin{array}{c}\text { ETR } \\
(\mathbf{m m})\end{array}$ & $\begin{array}{c}\text { Excedente } \\
(\mathbf{m m})\end{array}$ & $\begin{array}{c}\text { Excedente } \\
(\mathbf{M M C})\end{array}$ & $\begin{array}{c}\boldsymbol{\eta} \\
(\mathbf{m m})\end{array}$ & $\begin{array}{c}\boldsymbol{\eta} \\
\%\end{array}$ & $\begin{array}{c}\text { Estatus } \\
\text { IRI/ENSO }^{*}\end{array}$ \\
\hline $2000-2001(3)$ & 735.5 & 489.9 & 257.2 & 54.1 & -11.6 & -1.6 & Niño \\
$2001-2002(3)$ & 814.1 & 499.5 & 315.9 & 66.5 & -1.2 & -0.2 & Niño \\
$2002-2003(3)$ & 1088.9 & 600.0 & 449.2 & 99.6 & 39.7 & 3.6 & Niño-Neutral \\
$2003-2004(5)$ & 1009.2 & 674.8 & 372.3 & 78.4 & -37.9 & -3.8 & Neutral \\
$2004-2005(5)$ & 1436.9 & 656.4 & 709.7 & 149.4 & 70.8 & 4.9 & Niño-Neutral \\
$2005-2006(5)$ & 990.6 & 628.9 & 325.9 & 68.6 & 35.8 & 3.6 & Neutral-Niño \\
$2006-2007(5)$ & 1272.8 & 721.3 & 493.2 & 103.8 & 58.2 & 4.6 & Neutral \\
$2007-2008(5)$ & 1091.2 & 629.2 & 523.6 & 110.2 & -61.6 & -5.6 & Niña-Neutral \\
$2008-2009(5)$ & 1084.6 & 544.1 & 462.6 & 97.4 & 78.0 & 7.2 & Neutral-Niño \\
$2009-2010(5)$ & 2329.1 & 757.6 & 1028.3 & 216.5 & 543.2 & 23.3 & Niño-Niña \\
$2010-2011(5)$ & 1159.3 & 613.3 & 461.7 & 98.2 & 84.3 & 7.3 & Niña-Neutral \\
$2011-2012(5)$ & 1345.3 & 679.0 & 613.5 & 129.1 & 52.8 & 3.9 & Niña-Neutral \\
$2012-2013(4)$ & 1261.9 & 712.6 & 489.2 & 102.9 & 60.2 & 4.8 & Neutral \\
$2013-2014(4)$ & 950.4 & 686.5 & 248.1 & 52.2 & 15.8 & 1.7 & Neutral \\
$2014-2015(4)$ & 1115.6 & 598.2 & 439.0 & 92.4 & 78.4 & 7.0 & Niño \\
$2015-2016(4)$ & 1202.8 & 813.2 & 382.7 & 80.4 & 6.8 & 0.6 & Niño \\
\hline
\end{tabular}

$\eta=$ discrepancia, $\mathrm{ETR}=$ Evapotranspiración real, $\mathrm{MMC}=$ Millones de metros cúbicos.

La producción de agua varió de acuerdo con el fenómeno ENOS. Por ejemplo, por influencia del fenómeno El Niño, la producción de agua fue en promedio de aproximadamente 73.4 millones de metros cúbicos (MMC), tomando aquellos años donde este fenómeno predomino más. Mientras que, en los años mezcla (Neutral/Niño y Niño/Neutral), la producción promedio fue de aproximadamente 103,8 MMC. Durante los años Neutrales la producción de agua fue en promedio de 84,3 MMC, mientras en los años mezcla con condiciones de Neutral/Niña la producción de agua fue en promedio de 112,5 MMC y durante el único año mezcla Niño/Niña fue en 2010 y las precipitaciones se duplicaron respecto al promedio anual observado durante el resto de los años hidrológicos, por lo que la producción o excedente fue de 216.5 MMC, casi duplica otros años.

\begin{tabular}{|c|c|c|}
\hline 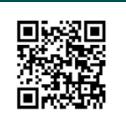 & (c) (i) () () & 129 \\
\hline
\end{tabular}




\section{Revista de CIENCIAS AMBIENTALES Tropical Journal of Environmental Sciences}

Revista de Ciencias Ambientales (Trop J Environ Sci) e-ISSN: 2215-3896

(Enero-Junio, 2021) . Vol 55(1): 118-142 DOI: https://doi.org/10.15359/rca.55-1.6

Open Access: www.revistas.una.ac.cr/ambientales e-mail: revista.ambientales@una.ac.cr Rodríguez-Cruz G.

Con lo cual se puede inferir que la variabilidad climática, dependiendo del fenómeno ENOS que se presente y su intensidad, afecta aumentando o disminuyendo la producción de agua de la subcuenca. La intensidad de estos fenómenos es medida de acuerdo con predicciones de la temperatura de la superficie del océano Pacifico, según los informes mensuales de la NOAA. También, el excedente producido por la subcuenca del río Guacerique durante la estación lluviosa y según los balances hídricos, se demuestra que sería capaz de suplir por sí sola la actual capacidad de almacenamiento anual de agua potable de la ciudad de Tegucigalpa de $55 \mathrm{MMC}$ de los tres principales reservorios, aun en presencia o influencia del fenómeno de El Niño (menores precipitaciones). Mientras en años neutrales y mezclas, la capacidad de producción de agua de la subcuenca del río Guacerique es incluso capaz de producir $110 \mathrm{MMC}$ y suplir la necesidad hídrica de la ciudad de Tegucigalpa de acuerdo con el estudio realizado por el SANAA de 1998, donde menciona que esta ciudad necesita entre 100 y 110 MMC.

Los balances hídricos históricos muestran que la producción de agua de la subcuenca del río Guacerique es suficiente para la ciudad de Tegucigalpa, y para que esta no sufra de fuertes racionamientos. Actualmente en Tegucigalpa el suministro de agua es de 12 horas a la semana para la mayoría de las colonias y barrios; en lugares más favorecidos, el suministro se duplica, mientras en otros lugares menos favorecidos, por la topografía de la ciudad, deben esperar entre 15 y 30 días para ser abastecidos. Sin embargo, una de las limitantes es la poca capacidad de embalsamiento de Los Laureles, con solo $10 \mathrm{MMC}$, capacidad que se ve reducida año tras año, debido a la sedimentación. Cuando se tienen excesos, estos se pierden al desbordarse y, además, no se cuenta con suficiente infraestructura que permita también aumentar la capacidad de potabilización y almacenamiento de estas grandes cantidades de agua en la época lluviosa. Cada año se realizan labores de dragado para aumentar el tiempo de vida del embalse, aunque al momento de este estudio no se habían realizado estudios de tasa de sedimentación y batimetría.

Los años hidrológicos de 2008, 2010, 2011 y 2012 fueron los únicos donde se presentó el fenómeno de La Niña y la producción hídrica se incrementó respecto a los años Niño, Neutral y Mezcla. En el año 2010, el aumento de las precipitaciones fue evidente, con la presencia de La Niña registró hasta $2500 \mathrm{~mm}$ de lluvia en las estaciones meteorológicas de la zona alta de la subcuenca, según el reporte histórico, respecto a los $1350 \mathrm{~mm}$ que normalmente caen en otros años, es decir, de un aumento de hasta $93 \%$, motivo por el cual se explica una discrepancia de $23.3 \%$. El año 2010 estuvo marcado por las inundaciones, deslizamientos y otros efectos colaterales causados por el exceso de humedad en los suelos de la zona que poseen pendientes de $15 \%$ y $30 \%$.

Respecto a los años neutrales, se nota una mejoría en el aumento de las precipitaciones respecto a los años Niño, mientras que los años mezcla Niño/Neutral en promedio registraron una producción de agua mayor que los dos anteriores. Sin embargo, se observó, durante los balances hídricos anuales, que con la entrada del fenómeno de El Niño disminuyeron las precipitaciones y con ello se redujo la disponibilidad de agua, con excepción del año 2015, pues cuando ocurrió el fenómeno de El Niño las precipitaciones aumentaron. Lo anterior puede ser una evidencia

\begin{tabular}{|c|c|c|}
\hline 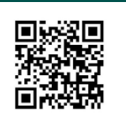 & (c) (i) () () & 130 \\
\hline
\end{tabular}




\section{Revista de CIENCIAS AMBIENTALES Tropical Journal of Environmental Sciences}

Revista de Ciencias Ambientales (Trop J Environ Sci) e-ISSN: 2215-3896

(Enero-Junio, 2021) . Vol 55(1): 118-142 DOI: https://doi.org/10.15359/rca.55-1.6

Open Access: www.revistas.una.ac.cr/ambientales e-mail: revista.ambientales@una.ac.cr Rodríguez-Cruz G.

más de la variabilidad climática, al parecer el fenómeno del Niño de 2015 tuvo una influencia moderada en la subcuenca Guacerique. Sin embargo, se rescata el hecho de que el estatus del IRI/ENSO es de corte global, lo que también dificulta la interpretación para regiones pequeñas como una subcuenca, donde los efectos de esta variabilidad climática pueden ser más severos o más benevolentes que en otras regiones.

Por ejemplo, para el año hidrológico 2015 - 2016, de acuerdo con el estatus IRI/ENSO, empezó el fenómeno del Niño para los meses de octubre, noviembre, diciembre de 2015 y enero de 2016 y se caracterizó por ser un Niño con afectación fuerte. El efecto de El Niño para Honduras significó aumento de temperaturas medias (Apéndices 6 al 10) y disminución de precipitaciones. Todas las variables atmosféricas apoyaron fuertemente los patrones de El Niño y el consenso de los modelos de predicciones del fenómeno ENOS indicó una continuación de las condiciones de El Niño fuerte durante esos cuatro meses en mención. Para los meses de octubre, diciembre de 2015 y enero de 2016, mostró una anomalía en las precipitaciones con una disminución de entre cinco (5) y hasta $70 \mathrm{~mm}$, dependiendo de la estación meteorológica y el mes (Apéndices 1 al 5).

\subsection{Transporte de sedimentos}

Para la interpretación de los resultados se utilizaron los datos de precipitación disponible de las estaciones meteorológicas del Servicio Autónomo Nacional de Acueductos y Alcantarillados (SANAA), el cual es un promedio para el mes de junio de tres (3) estaciones. Por lo tanto, la precipitación, los caudales promedios y la estimación de la carga de sedimentos corresponden al mes de junio de 2016. Los datos de caudal fueron un promedio de los datos de aforos realizados in situ y los datos históricos proporcionados por el SANAA (12 años) solo para el mes de junio. Con lo cual se hizo una relación entre la precipitación, el caudal del río y lo encontrado en las trampas instaladas. Los datos presentados a continuación están basados en los cálculos de los sedimentos atrapados y representa únicamente una idea general del transporte de estos mismos para el mes de junio 2016 y no para el año hidrológico completo.

A continuación se muestran los resultados de la cantidad de sedimentos que aportó cada uno de los tributarios y el río principal, de acuerdo con las trampas instaladas (Cuadro 6). Se hace la aclaración de que solo se pudieron obtener resultados de cinco (5) trampas, ya que una fue sustraída de su lugar por sujetos desconocidos y la otra trampa se dañó durante el transporte al laboratorio.

\begin{tabular}{|c|c|c|c|c|c|}
\hline 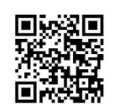 & (c) (1) () (2) & $\underset{\text { AMBENENALIES }}{\infty}$ & $\frac{1 \%}{\text { euna }}$ & 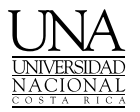 & 131 \\
\hline
\end{tabular}




\section{Revista de CIENCIAS AMBIENTALES Tropical Journal of Environmental Sciences}

Revista de Ciencias Ambientales (Trop J Environ Sci) e-ISSN: 2215-3896

(Enero-Junio, 2021) . Vol 55(1): 118-142 DOI: https://doi.org/10.15359/rca.55-1.6

Open Access: www.revistas.una.ac.cr/ambientales e-mail: revista.ambientales@una.ac.cr Rodríguez-Cruz G.

Cuadro 5. Resultados de trampas de sedimentos, estimación del aporte de sedimentos por parte de los tributarios y el río principal para el mes de junio 2016.

Table 5. Results of the sediment traps, contribution estimation of sediments for each tributary and principal river for June 2016.

\begin{tabular}{lcc}
\hline \multicolumn{1}{c}{$\begin{array}{c}\text { Precipitación Promedio junio } \mathbf{2 0 1 6} \\
(\mathbf{1 6 5 . 5} \mathbf{~} \mathbf{m m})\end{array}$} & $\begin{array}{c}\text { Caudal aproximado } \\
(\mathbf{m} 3 \text { día-1)* }\end{array}$ & $\begin{array}{c}\text { Estimación de arrastre de sedimentos para } \\
\text { junio (kg mes-1) }\end{array}$ \\
\hline Trampas & 57000 & 8.1 \\
(T6) Río Mateo & 140000 & 80.0 \\
(T5) Confluencia (Guacerique - Mateo) & 29000 & 12.2 \\
(T4) Quebrada El Ocotal & 29000 & 36.7 \\
(T2) Quebrada Achiote & 300000 & 488.8 \\
(T1) Batallón (río Guacerique) & & \\
\hline
\end{tabular}

* Caudal estimado del SANAA.

${ }^{* *}$ (T1) Batallón representa el total del arrastre por el río principal y sus tributarios.

Se observó que la trampa del batallón (T1) fue la que atrapó más sedimentos a través del caudal, que ingresó y pasó a través del tubo, respecto a los otras. Esto, debido a que donde se encuentra ubicada es la suma de todos los tributarios y se ubica en la parte baja de la subcuenca, próxima a entrar al embalse Los Laureles. Según los cálculos, esta trampa representa el $72 \%$ del total de sedimentos para el mes de junio de 2016 (Figura 3). La trampa colocada en el Río Mateo (T6) fue la que menos sedimentos atrapó, sorprende de manera particular, pues este río es el máximo tributario para el río principal, por lo cual se puede interpretar que esa parte de la subcuenca se encuentra en mejores condiciones, al poseer una mayor cobertura boscosa, lo que puede representar menor erosión hídrica.

La trampa que reportó el segundo lugar en cantidades de sedimentos fue la colocada en la confluencia (T5) entre el río Guacerique y el río Mateo; sin embargo, se pudo observar que la contribución del río Mateo es poca, solo el $1.7 \%$ del aporte (T6). Se interpreta que la mayor cantidad de arrastre de sedimentos ocurre a través del cauce del río Guacerique, que en este punto es de $16.3 \%$. La trampa de sedimento de la quebrada El Ocotal (T4) representó $2.5 \%$ de los sedimentos aportados al sistema, mientras que la trampa colocada en la quebrada El Achiote (T2) representó $7.5 \%$ del total del sedimento atrapado.

Sin embargo, esta cantidad representa únicamente una estimación general del transporte de sedimentos por la red de drenaje y no proporciona una cifra exacta de toda la cantidad de sedimentos que puede llegar hasta el embalse. Con la trampa no es posible estimar, por ejemplo, las cantidades que se depositan en las sinuosidades (vega) del mismo río, por diferencia de flujos o el ancho del caudal.

\begin{tabular}{|c|c|c|c|c|c|}
\hline 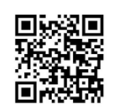 & (c) (1) () (2) & $\underset{\text { AMBENENALIES }}{\infty}$ & $\frac{1 \%}{\text { euna }}$ & 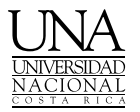 & 132 \\
\hline
\end{tabular}




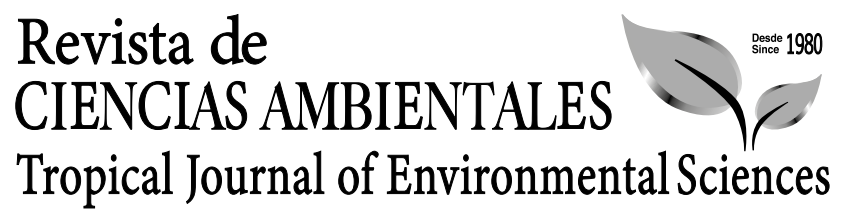

Revista de Ciencias Ambientales (Trop J Environ Sci) e-ISSN: 2215-3896 (Enero-Junio, 2021) . Vol 55(1): 118-142 DOI: https://doi.org/10.15359/rca.55-1.6 Open Access: www.revistas.una.ac.cr/ambientales e-mail: revista.ambientales@una.ac.cr Rodríguez-Cruz G.

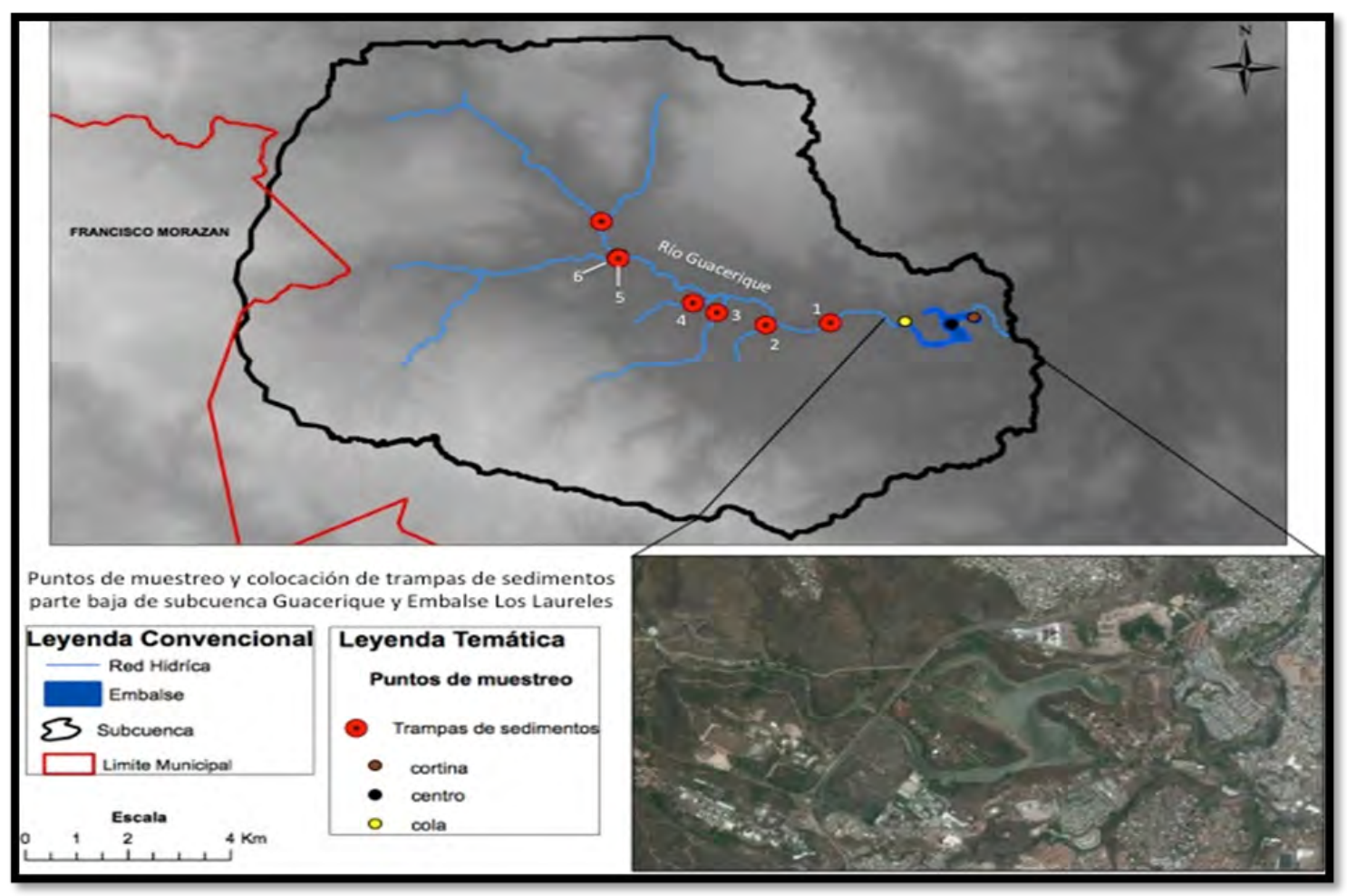

Figura 4. Aporte porcentual estimado del arrastre de sedimentos de acuerdo con las trampas para junio de 2016. Figure 4. Estimate percentage contributions of the drag sediments according to the traps in June 2016.

\section{Conclusiones}

La distribución de la precipitación en la subcuenca del río Guacerique es irregular, debido a la zonificación en función de la altura, vegetación, cercanía del embalse y variabilidad climática producida por el fenómeno ENOS, que afecta a la región en particular. Sin embargo, a pesar de estas diferencias en precipitación entre años hidrológicos durante la serie de años (2001 - 2016), en general no se observa una tendencia a la disminución de la precipitación histórica promedio acumulada; al contrario, aparece un ligero aumento de entre $1 \%$ y $2 \%$ de esa precipitación histórica promedio acumulada. Sin embargo, se pueden observar, de manera general, diferencias en las precipitaciones y la ETR entre años, dependiendo de la intensidad del fenómeno ENOS, para lo cual se debe contar con mayor infraestructura de almacenamiento para minimizar el impacto de años con menor precipitación, y evitar, con ello, la escasez y racionamientos.

En cuanto al balance hídrico histórico consolidado, la producción de agua en la subcuenca Guacerique es de $248 \mathrm{MMC}$, y, más de la mitad de esa producción de la subcuenca del río Guacerique, se evapotranspira (57 \%) y el restante (43\%) es el excedente histórico unos 99.4 MMC; sin embargo, se puede observar que este excedente varió cada año entre 52 y 216 MMC. Esta

\begin{tabular}{|c|c|c|}
\hline 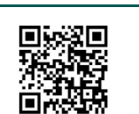 & (c) (1) (8) () () & 133 \\
\hline
\end{tabular}




\section{Revista de CIENCIAS AMBIENTALES Tropical Journal of Environmental Sciences}

Revista de Ciencias Ambientales (Trop J Environ Sci) e-ISSN: 2215-3896

(Enero-Junio, 2021) . Vol 55(1): 118-142 DOI: https://doi.org/10.15359/rca.55-1.6

Open Access: www.revistas.una.ac.cr/ambientales e-mail: revista.ambientales@una.ac.cr Rodríguez-Cruz G.

producción puede ser suficiente para abastecer al $30 \%$ de la población de Tegucigalpa al menos cada 24 horas; sin embargo, esta se pierde, en gran medida, debido a la poca capacidad de embalsamiento, potabilización y almacenaje del agua tratada, por lo que la ciudad de Tegucigalpa sufre de fuertes racionamientos en la época seca y, ahora, en la época lluviosa.

Los resultados de las trampas de sedimentos mostraron que el principal aporte proviene del río Guacerique, el cual es evidente en la zona baja de la subcuenca Guacerique con mayor intervención antrópica. Por otro lado, el aporte de los tres tributarios en el estudio es poco significativo. Respecto al tipo de trampas, se encontró que la metodología utilizada (tipo de trampas) para la subcuenca del río Guacerique puede ser menos eficiente comparada con otras, aunque su principal ventaja radica en su bajo costo (económico) y la facilidad de transporte y colocación. Para caudales bajos se podría adaptar la metodología o usar una diferente, para minimizar el margen de error, el cual puede ser alto, al no reflejar con precisión el movimiento de los sedimentos. Se recomienda, además, hacer mediciones durante un año hidrológico completo.

Finalmente, la sedimentación producto del arrastre a través de la red hídrica puede estar contribuyendo a una disminución de la capacidad de embalsamiento de acuerdo con la estimación de las trampas para el mes de junio 2016. Por tanto, se hace urgente y necesario el estudio completo de tasas de sedimentación y batimetría en el embalse. La sedimentación puede estar siendo intensificada, producto de la deforestación acelerada de la subcuenca Guacerique, la urbanización en las proximidades del embalse y su red hídrica. También, con evidencias observadas en el balance hídrico, la variabilidad climática, pendiendo de la intensidad del fenómeno ENOS, puede provocar disminución o aumento de la disponibilidad del agua. Dependerá del ente administrador aprovechar los volúmenes cuando se den excesos, para poder embalsarlos, almacenarlos y potabilizarlos.

\section{5. Ética y conflicto de intereses}

El autor declara que ha cumplido totalmente con todos los requisitos éticos y legales pertinentes, tanto durante el estudio como en la producción del manuscrito; que no hay conflictos de intereses de ningún tipo; que todas las fuentes financieras se mencionan completa y claramente en la sección de agradecimientos; y que está totalmente de acuerdo con la versión final editada del artículo.

\section{Agradecimientos}

El autor expresa un especial agradecimiento a la Dra. Lourdes Reyes de Nasser, por su apoyo y confianza incondicional a la investigación científica; también para el Ing. Roque Andrade, por el apoyo logístico y de gestión, ambos del Servicio Autónomo Nacional de Acueductos y Alcantarillados (SANAA). Al Instituto Hondureño de Ciencias de la Tierra, por el préstamo de equipo y asesoría a través del MSc. Maynor Ruiz. A la MSc. Yelba Flores, por sus consejos en el uso de la metodología de balances hídricos. También al Centro de Investigación para los

\begin{tabular}{|c|c|c|}
\hline 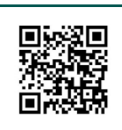 & (c) (i) () () & 134 \\
\hline
\end{tabular}




\section{Revista de CIENCIAS AMBIENTALES Tropical Journal of Environmental Sciences}

Revista de Ciencias Ambientales (Trop J Environ Sci) e-ISSN: 2215-3896 (Enero-Junio, 2021) . Vol 55(1): 118-142 DOI: https://doi.org/10.15359/rca.55-1.6 Open Access: www.revistas.una.ac.cr/ambientales e-mail: revista.ambientales@una.ac.cr Rodríguez-Cruz G.

Recursos Acuáticos de Nicaragua, CIRA-UNAN Managua, por el apoyo logístico, de asesoría y equipo. Además, a las personas revisoras anónimas de la Revista, por sus aportes, los cuales enriquecieron el presente documento.

\section{Referencias}

Bates, B. C., Z. W. Kundzewicz, S. Wu y J. P. Palutikof, Eds., (2008). El cambio climático y el agua. Documento técnico del Grupo Intergubernamental de Expertos sobre el Cambio ClimáticoSecretaría del IPCC.

Berrios, S. (2014). Protección legal de las fuentes de agua en la ciudad de Tegucigalpa. Revista de Derecho, 35(1). https://doi.org/10.5377/lrd.v35i0.1784

Bouraoui, F., Grizzetti, B., Granlund, K., Rekolainen, S. y Bidoglio, G., (2004). Impact of climate change on the water cycle and nutrient losses in a Finnish catchment. Climatic Change, 66, 109-126. https://doi.org/10.1023/B:CLIM.0000043147.09365.e3

Fattorelli, S., y Fernández, P. (2011). Diseño hidrológico (2 ${ }^{\text {da }}$ Ed.). Editorial WASA-GN. https:// marianiztli.files.wordpress.com/2016/06/diseno-hidrologico.pdf

INE. (2015). Proyecciones de población 2014 - 2030. Instituto Nacional de Estadísticas de Honduras.

International Research Institute (IRI). (2016). IRI ENSO Forecast, 2016 december quick look. https://www.iri.columbia.edu/our-expertise/climate/forecast/enso/current/

IPCC (Intergovernmental Panel on Climate Change). (2007). Climate Change 2007: The Physical Science Basis: Summary for Policymakers. Fourth Assessment Report of the IPCC (ed. by M. L. Parry, O. F. Canziani, J. P. Palutikof, P. J. van der Linden y C. E. Hanson). Cambridge University Press.

Leemans, R., y Kleidon, A. (2002). Regional and global assessment of the dimensions of desertification. (En J. F. Reynold and D.bS. Smith (Eds.), Global Desertification: Do ans Cause Deserts? (pp. 215 - 232). Dahlem University Press.

López, R. (1990). El diagnóstico de suelos y plantas. Método de campo y laboratorio (4. ${ }^{\text {ta }}$ ed.). Editorial Madrid Mundi-Prensa.

Mimikou, M., Blatas, E., Varanaou, E. y Pantazis, K. (2000). Regional impacts of climate change on water resources quantity and quality indicators. J. Hydrol., 234, 95 - 109. https://doi. org/10.1016/S0022-1694(00)00244-4

National Oceanic and Atmospheric Administration (NOAA). (2016). Physical Sciences Division, Boulder Colorado. https://www.esrl.noaa.gov/psd

\begin{tabular}{|c|c|c|}
\hline 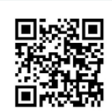 & (c) (1) (9) & 135 \\
\hline
\end{tabular}




\section{Revista de CIENCIAS AMBIENTALES Tropical Journal of Environmental Sciences}

Revista de Ciencias Ambientales (Trop J Environ Sci) e-ISSN: 2215-3896

(Enero-Junio, 2021) . Vol 55(1): 118-142 DOI: https://doi.org/10.15359/rca.55-1.6 Open Access: www.revistas.una.ac.cr/ambientales e-mail: revista.ambientales@una.ac.cr Rodríguez-Cruz G.

Neff, R., Chang, H., Knight, C., Najjar, R., Yarnal, B., y Walker, H. (2000). Impact of climate variation and change on Mid-Atlantic Region hydrology and water resources. Climate Res., 14, 207 - 218. https://doi.org/10.3354/cr014207

Organización Meteorológica Mundial. (2018). El Niño/La Niña hoy. http://www.wmo.int/pages/ prog/wcp/wcasp/LC-LRFMME/index.php

PREPAC. (2005). Inventario regional de los cuerpos de agua continentales del istmo centroamericano. Plan Regional de Pesca y Agricultura Continental. http://www.oirsa.org/aplicaciones/ subidoarchivos/BibliotecaVirtual/EmbalsesHonduras.pdf

SANAA. (2004). Planta de tratamiento de agua Luis Salvador Úlloa (Los Laureles) http://www. sanaa.hn/leytransparencia/PDFs/ESTRUCTURA\%20ORGANICA\%20Y\%20SERVICIOS/ ATRIBUICIONES\%20X\%20UNIDAD\%20ADMINISTRATIVA/Planta\%20de\%20Tratamiento\%20de\%20Agua\%20Luis\%20Salvador\%20Ulloa\%20-\%20Los\%20Laureles.pdf

Sokolov, A. A., y Chapman, T. G. (1981). Métodos de cálculo de balance hídrico: Guía internacional de investigación y métodos. Instittuto de hidrología de España / UNESCO,

Thornthwaite, C. W. (1948). An approach towards a rational classification of climate. Geogr.

Rev., 38, 55-89. https://doi.org/10.2307/210739

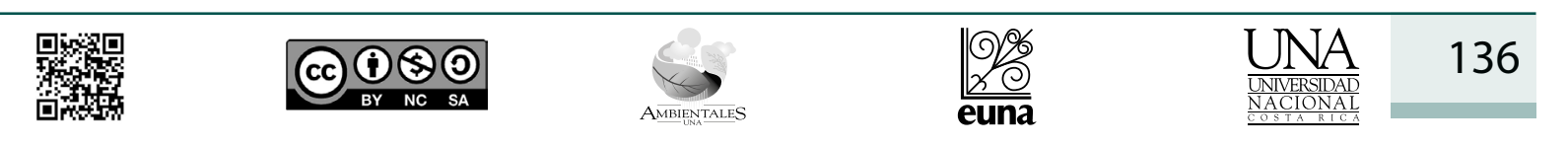




\section{Revista de CIENCIAS AMBIENTALES Tropical Journal of Environmental Sciences}

Revista de Ciencias Ambientales (Trop J Environ Sci) e-ISSN: 2215-3896

(Enero-Junio, 2021) . Vol 55(1): 118-142 DOI: https://doi.org/10.15359/rca.55-1.6 Open Access: www.revistas.una.ac.cr/ambientales e-mail: revista.ambientales@una.ac.cr Rodríguez-Cruz G.

\section{Apéndices}

Apéndice 1. Consolidado mensual periodo 2001 al 2016, estación \#1, "El Batallón". Appendix 1. Monthly consolidated to 2001 to 2016, station \#1, "El Batallón"

\begin{tabular}{cccccccccccccc}
\hline Fecha & Oct & Nov & Dic & Ene & Feb & Mar & Abr & May & Jun & Jul & Ago & Sep & Total \\
\hline $2000-2001$ & 70.7 & 7.5 & 10.5 & 4.0 & 0.0 & 6.5 & 11.0 & 204.8 & 13.0 & 54.9 & 127.8 & 178.7 & 689.4 \\
$2001-2002$ & 71.2 & 8.0 & 5.3 & 0.5 & 7.0 & 0.0 & 0.0 & 153.7 & 199.7 & 66.9 & 52.3 & 158.0 & 722.6 \\
$2002-2003$ & 41.6 & 13.6 & 19.5 & 0.2 & 0.0 & 18.3 & 19.2 & 261.9 & 227.2 & 85.1 & 115.1 & 123.5 & 925.2 \\
$2003-2004$ & 154.9 & 50.7 & 0.0 & 4.1 & 16.7 & 0.2 & 109.2 & 74.2 & 58.1 & 105.9 & 34.0 & 205.5 & 813.5 \\
$2004-2005$ & 107.5 & 34.4 & 8.7 & 0.0 & 2.5 & 36.6 & 50.1 & 256.6 & 251.1 & 226.9 & 149.0 & 139.0 & 1262.4 \\
$2005-2006$ & 91.8 & 11.1 & 1.9 & 10.0 & 0.5 & 0.0 & 67.2 & 110.0 & 230.9 & 111.5 & 56.7 & 103.9 & 795.5 \\
$2006-2007$ & 141.6 & 83.0 & 21.0 & 2.2 & 1.9 & 15.5 & 80.5 & 72.1 & 148.5 & 34.8 & 270.1 & 148.8 & 1020.0 \\
$2007-2008$ & 106.3 & 32.7 & 0.0 & 2.3 & 27.2 & 1.8 & 27.9 & 106.7 & 85.0 & 156.7 & 165.3 & 203.1 & 915.0 \\
$2008-2009$ & 228.8 & 13.4 & 5.0 & 23.9 & 2.3 & 2.9 & 7.0 & 191.3 & 250.2 & 88.9 & 42.2 & 61.9 & 917.8 \\
$2009-2010$ & 85.6 & 56.3 & 31.9 & 3.2 & 5.8 & 0.0 & 192.4 & 314.1 & 145.8 & 229.3 & 340.2 & 252.2 & 1656.8 \\
$2010-2011$ & 14.5 & 10.6 & 0.0 & 2.2 & 2.8 & 2.8 & 59.0 & 174.2 & 190.9 & 165.7 & 67.6 & 189.6 & 879.9 \\
$2011-2012$ & 165.2 & 44.8 & 1.7 & 0.1 & 4.0 & 2.0 & 139.5 & 225.4 & 92.6 & 23.2 & 232.5 & 46.7 & 977.7 \\
$2012-2013$ & 202.0 & 2.3 & 0.0 & 5.6 & 0.0 & 14.2 & 25.4 & 160.8 & 121.7 & 61.2 & 178.3 & 226.4 & 997.9 \\
$2013-2014$ & 152.6 & 35.1 & 31.4 & 7.0 & 2.3 & 14.9 & 71.1 & 97.7 & 169.8 & 28.7 & 104.7 & 103.5 & 818.8 \\
$2014-2015$ & 192.4 & 17.7 & 15.9 & 1.1 & 1.3 & 4.5 & 58.5 & 45.9 & 291.8 & 27.0 & 20.5 & 227.5 & 904.1 \\
$2015-2016$ & 170.6 & 55.2 & 6.6 & 0.0 & 0.0 & 32.4 & 10.8 & 206.7 & 165.5 & 77.8 & 207.1 & 164.5 & 1097.2 \\
\hline Promedio & $\mathbf{1 2 4 . 8}$ & $\mathbf{2 9 . 8}$ & $\mathbf{1 0 . 0}$ & $\mathbf{4 . 2}$ & $\mathbf{4 . 6}$ & $\mathbf{9 . 5}$ & $\mathbf{5 8 . 1}$ & $\mathbf{1 6 6 . 0}$ & $\mathbf{1 6 5 . 1}$ & $\mathbf{9 6 . 5}$ & $\mathbf{1 3 5 . 2}$ & $\mathbf{1 5 8 . 3}$ & $\mathbf{9 6 2 . 1}$ \\
\hline & & & & & & & & & & & & & \\
\hline
\end{tabular}

Apéndice 2. Consolidado mensual periodo 2001 al 2016, estación \#2, "Quiebramontes".

Appendix 2. Monthly consolidated to 2001 to 2016, station \#2, "Quiebramontes"

\begin{tabular}{cccccccccccccc}
\hline Fecha & Oct & Nov & Dic & Ene & Feb & Mar & Abr & May & Jun & Jul & Ago & Sep & Total \\
\hline $2000-2001$ & 93.0 & 29.1 & 1.4 & 14.0 & 0.4 & 35.4 & 49.0 & 171.8 & 8.4 & 53.7 & 187.5 & 144.7 & 788.4 \\
$2001-2002$ & 103.8 & 6.3 & 11.9 & 0.7 & 7.1 & 1.0 & 9.5 & 149.5 & 198.4 & 82.3 & 62.7 & 187.7 & 820.9 \\
$2002-2003$ & 105.6 & 16.5 & 3.1 & 3.8 & 0.8 & 12.8 & 23.1 & 289.4 & 251.1 & 85.3 & 135.2 & 132.9 & 1059.6 \\
$2003-2004$ & 154.6 & 32.3 & 0.0 & 0.4 & 19.9 & 4.2 & 92.3 & 97.8 & 48.8 & 186.8 & 47.4 & 127.9 & 812.4 \\
$2004-2005$ & 129.3 & 27.6 & 3.7 & 3.1 & 6.0 & 35.0 & 17.2 & 219.9 & 297.6 & 160.6 & 99.2 & 163.0 & 1162.2 \\
$2005-2006$ & 93.1 & 17.4 & 8.1 & 9.7 & 0.4 & 0.0 & 28.4 & 129.9 & 301.2 & 150.6 & 116.3 & 139.2 & 994.3 \\
$2006-2007$ & 253.2 & 91.4 & 31.1 & 2.4 & 5.6 & 12.6 & 66.0 & 195.6 & 205.7 & 84.4 & 268.3 & 234.1 & 1450.4 \\
$2007-2008$ & 140.5 & 38.8 & 0.4 & 5.4 & 20.6 & 1.0 & 24.6 & 107.8 & 127.9 & 179.9 & 191.2 & 197.4 & 1035.5 \\
$2008-2009$ & 259.8 & 11.2 & 4.9 & 12.3 & 3.8 & 2.1 & 1.3 & 209.2 & 311.1 & 88.3 & 55.4 & 68.0 & 1027.4 \\
$2009-2010$ & 59.6 & 82.3 & 58.8 & 3.2 & 3.3 & 0.0 & 194.1 & 345.8 & 218.4 & 237.3 & 439.0 & 297.9 & 1939.7
\end{tabular}




\section{Revista de CIENCIAS AMBIENTALES Tropical Journal of Environmental Sciences}

Revista de Ciencias Ambientales (Trop J Environ Sci) e-ISSN: 2215-3896 (Enero-Junio, 2021) . Vol 55(1): 118-142 DOI: https://doi.org/10.15359/rca.55-1.6 Open Access: www.revistas.una.ac.cr/ambientales e-mail: revista.ambientales@una.ac.cr Rodríguez-Cruz G.

\begin{tabular}{cccccccccccccc}
\hline Fecha & Oct & Nov & Dic & Ene & Feb & Mar & Abr & May & Jun & Jul & Ago & Sep & Total \\
\hline $2010-2011$ & 18.4 & 43.7 & 2.6 & 2.9 & 0.9 & 2.9 & 110.0 & 298.2 & 175.5 & 188.0 & 106.6 & 262.1 & 1211.8 \\
$2011-2012$ & 158.1 & 46.9 & 2.5 & 2.1 & 5.4 & 5.7 & 130.0 & 452.0 & 140.2 & 51.1 & 208.9 & 102.7 & 1305.6 \\
$2012-2013$ & 259.9 & 14.4 & 22.2 & 14.6 & 0.0 & 31.2 & 38.3 & 135.0 & 164.8 & 104.8 & 250.0 & 315.7 & 1350.9 \\
$2013-2014$ & 148.0 & 42.7 & 11.1 & 10.8 & 0.0 & 26.0 & 45.0 & 68.7 & 166.9 & 32.7 & 132.3 & 108.0 & 792.2 \\
$2014-2015$ & 110.6 & 17.6 & 15.4 & 2.9 & 0.0 & 0.0 & 36.5 & 42.6 & 244.6 & 31.4 & 21.0 & 344.7 & 867.3 \\
$2015-2016$ & 127.5 & 56.9 & 30.6 & 0.0 & 0.0 & 24.7 & 19.6 & 141.4 & 224.4 & 99.5 & 205.8 & 147.2 & 1077.6 \\
\hline Promedio & $\mathbf{1 3 8 . 4}$ & $\mathbf{3 5 . 9}$ & $\mathbf{1 3 . 0}$ & $\mathbf{5 , 5}$ & $\mathbf{4 . 6}$ & $\mathbf{1 2 . 2}$ & $\mathbf{5 5 . 3}$ & $\mathbf{1 9 0 . 9}$ & $\mathbf{1 9 2 . 8}$ & $\mathbf{1 1 3 . 5}$ & $\mathbf{1 5 7 . 9}$ & $\mathbf{1 8 5 . 8}$ & $\mathbf{1 1 0 6 . 0}$ \\
\hline
\end{tabular}

Apéndice 3. Consolidado mensual periodo 2004 al 2012, estación \#3, "El Escarbadero".

Appendix 3. Monthly consolidated to 2004 to 2012, station \#3, "El Escarbadero"

\begin{tabular}{cccccccccccccc}
\hline Fecha & Oct & Nov & Dic & Ene & Feb & Mar & Abr & May & Jun & Jul & Ago & Sep & Total \\
\hline $2003-2004$ & 104.5 & 41.6 & 1.7 & 9.3 & 43.7 & 22.8 & 113.8 & 223.0 & 156.2 & 283.0 & 106.3 & 117.3 & 1223.2 \\
$2004-2005$ & 148.2 & 52.5 & 14.7 & 7.2 & 5.0 & 25.4 & 12.5 & 340.4 & 417.8 & 239.6 & 150.4 & 249.8 & 1663.5 \\
$2005-2006$ & 87.6 & 37.5 & 13.3 & 29.3 & 8.4 & 0.0 & 59.6 & 114.9 & 374.9 & 159.2 & 87.7 & 177.6 & 1150 \\
$2006-2007$ & 170.6 & 97.7 & 37.6 & 10.9 & 11.0 & 19.1 & 84.6 & 152.4 & 187.7 & 66.3 & 199.8 & 325.0 & 1362.7 \\
$2007-2008$ & 163.4 & 31.1 & 4.0 & 5.6 & 6.6 & 3.4 & 40.4 & 116.0 & 112.7 & 242.9 & 230.6 & 180.2 & 1136.9 \\
$2008-2009$ & 286.9 & 11,0 & 13.6 & 13.2 & 15.9 & 2.0 & 0.0 & 189.0 & 312.6 & 94.7 & 81.1 & 76.8 & 1096.8 \\
$2009-2010$ & 42.6 & 75.2 & 52.3 & 7.7 & 11.7 & 0.0 & 165.6 & 383.5 & 324.7 & 281.3 & 772.3 & 409.3 & 2526.2 \\
$2010-2011$ & 10.0 & 0.0 & 0.0 & 5.8 & 2.8 & 28.9 & 24.3 & 81.8 & 144.8 & 249.0 & 275.1 & 171.5 & 994 \\
$2011-2012$ & 489.7 & 24.4 & 8.6 & 13.1 & 12.3 & 6.1 & 74.2 & 223.6 & 257.6 & 23.5 & 59.5 & 113.2 & 1305.8 \\
\hline Promedio & $\mathbf{1 6 7 . 1}$ & $\mathbf{4 1 . 2}$ & $\mathbf{1 6 . 2}$ & $\mathbf{1 1 . 3}$ & $\mathbf{1 3 . 0}$ & $\mathbf{1 2 . 0}$ & $\mathbf{6 3 . 9}$ & $\mathbf{2 0 2 . 7}$ & $\mathbf{2 5 4 . 3}$ & $\mathbf{1 8 2 . 2}$ & $\mathbf{2 1 8 . 1}$ & $\mathbf{2 0 2 . 3}$ & $\mathbf{1 3 8 4 . 3}$ \\
\hline
\end{tabular}

Apéndice 4. Consolidado mensual periodo 2004 al 2016, estación \#4, "Rincón de Dolores".

Appendix 4. Monthly consolidated to 2004 to 2016, station \#4, "Rincón de Dolores"

\begin{tabular}{cccccccccccccc}
\hline Fecha & Oct & Nov & Dic & Ene & Feb & Mar & Abr & May & Jun & Jul & Ago & Sep & Total \\
\hline $2003-2004$ & 172.0 & 62.4 & 3.5 & 22.8 & 36.2 & 54.7 & 123.4 & 160.1 & 127.3 & 104.4 & 74.2 & 157.7 & 1098.7 \\
$2004-2005$ & 117.7 & 85.1 & 27.0 & 106.9 & 9.1 & 42.0 & 52.2 & 326.1 & 437.9 & 175.4 & 221.3 & 195.6 & 1796.3 \\
$2005-2006$ & 63.4 & 55.0 & 27.9 & 67.0 & 10.6 & 0.7 & 54.9 & 108.3 & 289.8 & 228.0 & 121.5 & 54.6 & 1081.7 \\
$2006-2007$ & 76.8 & 88.1 & 36.1 & 16.6 & 16.0 & 56.7 & 49.3 & 79.6 & 189.1 & 94.2 & 249.9 & 329.4 & 1281.8 \\
$2007-2008$ & 212.9 & 68.2 & 16.2 & 17.0 & 13.6 & 5.0 & 49.9 & 127.9 & 165.7 & 314.1 & 223.0 & 188.5 & 1402 \\
$2008-2009$ & 362.3 & 4.6 & 6.4 & 53.1 & 9.2 & 1.1 & 57.0 & 232.9 & 304.3 & 54.3 & 97.6 & 81.6 & 1264.4 \\
$2009-2010$ & 98.4 & 76.1 & 58.4 & 13.6 & 15.2 & 1.2 & 312.2 & 362.4 & 273.3 & 277.0 & 692.1 & 344.1 & 2524 \\
$2010-2011$ & 10.7 & 12.4 & 5.1 & 12.8 & 53.7 & 25.8 & 31.7 & 176.2 & 221.8 & 343.2 & 136.2 & 329.5 & 1359.1 \\
$2011-2012$ & 204.2 & 51.2 & 35.2 & 30.8 & 21.4 & 15.7 & 121.6 & 386.2 & 168.2 & 98.4 & 255.1 & 145.9 & 1533.9 \\
$2012-2013$ & 206.1 & 54.0 & 54.3 & 56.2 & 1.5 & 73.8 & 51.1 & 147.9 & 109.7 & 194.1 & 109.0 & 276.5 & 1334.2 \\
$2013-2014$ & 153.7 & 66.0 & 79.1 & 76.0 & 35.4 & 14.7 & 12.5 & 126.4 & 141.7 & 54.7 & 195.5 & 307.6 & 1263.3
\end{tabular}




\section{Revista de CIENCIAS AMBIENTALES Tropical Journal of Environmental Sciences}

Revista de Ciencias Ambientales (Trop J Environ Sci) e-ISSN: 2215-3896 (Enero-Junio, 2021) . Vol 55(1): 118-142 DOI: https://doi.org/10.15359/rca.55-1.6 Open Access: www.revistas.una.ac.cr/ambientales e-mail: revista.ambientales@una.ac.cr Rodríguez-Cruz G.

\begin{tabular}{cccccccccccccc}
\hline Fecha & Oct & Nov & Dic & Ene & Feb & Mar & Abr & May & Jun & Jul & Ago & Sep & Total \\
\hline $2014-2015$ & 248.3 & 76.8 & 31.2 & 34.7 & 11.8 & 30.1 & 24.3 & 76.1 & 420.9 & 82.0 & 77.5 & 268.1 & 1381.8 \\
$2015-2016$ & 251.5 & 99.6 & 27.9 & 12.5 & 35.6 & 54.7 & 55.2 & 115.1 & 221.5 & 134.5 & 182.2 & 184.8 & 1375.1 \\
\hline Promedio & $\mathbf{1 6 7 . 5}$ & $\mathbf{6 1 . 5}$ & $\mathbf{3 1 . 4}$ & $\mathbf{4 0 . 0}$ & $\mathbf{2 0 . 7}$ & $\mathbf{2 8 . 9}$ & $\mathbf{7 6 . 6}$ & $\mathbf{1 8 6 . 6}$ & $\mathbf{2 3 6 . 2}$ & $\mathbf{1 6 5 . 7}$ & $\mathbf{2 0 2 . 7}$ & $\mathbf{2 2 0 . 3}$ & $\mathbf{1 4 3 8 . 2}$ \\
\hline
\end{tabular}

Apéndice 5. Consolidado mensual periodo 2001 al 2016, estación \#5, "San Matías".

Appendix 5. Monthly consolidated to 2001 to 2016, station \#5, "San Matías"

\begin{tabular}{cccccccccccccc}
\hline Fecha & Oct & Nov & Dic & Ene & Feb & Mar & Abr & May & Jun & Jul & Ago & Sep & Total \\
\hline $2000-2001$ & 101.6 & 9.2 & 17.8 & 3.9 & 7.0 & 13.5 & 0.7 & 134.1 & 38.7 & 77.9 & 103.3 & 220.9 & 728.6 \\
$2001-2002$ & 141.1 & 12.8 & 16.6 & 4.2 & 18.3 & 6.6 & 3.7 & 91.4 & 268.4 & 48.3 & 46.8 & 240.7 & 898.9 \\
$2002-2003$ & 239.1 & 19.7 & 20.2 & 16.8 & 9.3 & 12.7 & 17.0 & 233.0 & 345.2 & 89.8 & 126.5 & 152.6 & 1281.9 \\
$2003-2004$ & 115.4 & 70.5 & 16.6 & 31.7 & 42.3 & 41.2 & 108.8 & 95.3 & 161.3 & 222.5 & 62.8 & 129.9 & 1098.3 \\
$2004-2005$ & 134.4 & 48.7 & 17.8 & 16.0 & 5.4 & 22.6 & 9.4 & 234.5 & 390.1 & 131.5 & 173.4 & 116.3 & 1300.1 \\
$2005-2006$ & 110.7 & 61.9 & 16.9 & 16.4 & 9.9 & 2.6 & 31.0 & 161.7 & 209.8 & 102.0 & 111.2 & 97.3 & 931.4 \\
$2006-2007$ & 172.4 & 89.7 & 40.5 & 7.3 & 12.7 & 27.6 & 79.9 & 101.0 & 151.7 & 84.5 & 203.7 & 278.0 & 1249 \\
$2007-2008$ & 109.9 & 49.4 & 8.6 & 6.6 & 16.7 & 6.4 & 71.7 & 138.8 & 92.2 & 178.8 & 162.3 & 125.0 & 966.4 \\
$2008-2009$ & 252.0 & 10.3 & 12.0 & 25.0 & 12.4 & 0.0 & 5.1 & 190.6 & 367.9 & 93.4 & 85.6 & 62.4 & 1116.7 \\
$2009-2010$ & 81.5 & 51.7 & 54.9 & 8.8 & 19.7 & 0.0 & 193.1 & 395.3 & 438.1 & 472.5 & 804.3 & 479.0 & 2998.9 \\
$2010-2011$ & 12.4 & 53.3 & 4.1 & 32.9 & 8.4 & 10.2 & 91.4 & 159.3 & 149.3 & 280.2 & 179.9 & 370.2 & 1351.6 \\
$2011-2012$ & 167.9 & 31.5 & 25.0 & 33.7 & 28.8 & 20.4 & 118.1 & 336.8 & 122.2 & 82.3 & 327.2 & 309.5 & 1603.4 \\
$2012-2013$ & 264.8 & 24.8 & 49.4 & 57.7 & 10.3 & 101.3 & 19.3 & 118.3 & 171.2 & 136.0 & 143.7 & 267.9 & 1364.7 \\
$2013-2014$ & 48.8 & 49.2 & 29.0 & 39.4 & 20.7 & 47.5 & 29.0 & 110.3 & 141.3 & 35.5 & 85.7 & 290.7 & 927.1 \\
$2014-2015$ & 275.9 & 78.9 & 65.6 & 56.4 & 25.1 & 59.7 & 28.3 & 68.8 & 309.1 & 58.4 & 33.9 & 249.2 & 1309.3 \\
$2015-2016$ & 136.3 & 62.3 & 75.6 & 24.6 & 22.7 & 16.3 & 42.1 & 166.0 & 263.4 & 132.4 & 166.8 & 152.7 & 1261.2 \\
\hline Promedio & 148.8 & 45.2 & 29.4 & 23.8 & $\mathbf{1 6 . 9}$ & $\mathbf{2 4 . 3}$ & $\mathbf{5 3 . 0}$ & $\mathbf{1 7 1 . 0}$ & $\mathbf{2 2 6 . 2}$ & $\mathbf{1 3 9 . 1}$ & $\mathbf{1 7 6 . 1}$ & $\mathbf{2 2 1 . 4}$ & $\mathbf{1} 274.2$ \\
\hline & & & & & & & & & & & & &
\end{tabular}

Apéndice 6. Consolidado mensual periodo 2001 al 2016, estación \#1, "El Batallón”.

Appendix 6. Monthly consolidated to 2001 to 2016, station \#1, "El Batallón"

\begin{tabular}{ccccccccccccccc}
\hline Fecha & Oct & Nov & Dic & Ene & Feb & Mar & Abr & May & Jun & Jul & Ago & Sep & Prom/año \\
\hline $2000-2001$ & 22.2 & 22.3 & 20.8 & 21.0 & 22.2 & 23.2 & 24.3 & 24.2 & 23.5 & 23.5 & 23.6 & 22.3 & 22.8 \\
$2001-2002$ & 22.5 & 21.6 & 22.5 & 21.5 & 22.4 & 23.7 & 25.2 & 24.9 & 23.9 & 23.0 & 23.8 & 23.3 & 23.2 \\
$2002-2003$ & 23.2 & 21.8 & 22.1 & 20.5 & 23.3 & 24.4 & 24.7 & 25.4 & 23.7 & 23.5 & 23.5 & 23.7 & 23.3 \\
$2003-2004$ & 23.3 & 22.2 & 19.8 & 20.8 & 21.8 & 23.2 & 24.1 & 24.0 & 23.6 & 23.4 & 23.7 & 23.9 & 22.8 \\
$2004-2005$ & 23.4 & 21.6 & 21.2 & 21.0 & 22.8 & 25.2 & 25.6 & 24.2 & 23.9 & 23.5 & 23.8 & 23.1 & 23.3 \\
$2005-2006$ & 21.9 & 21.3 & 21.9 & 21.1 & 21.9 & 23.9 & 24.7 & 25.4 & 23.1 & 23.5 & 23.9 & 23.8 & 23.0 \\
$2006-2007$ & 23.6 & 21.4 & 21.9 & 22.3 & 23.0 & 23.8 & 25.6 & 25.4 & 24.2 & 24.3 & 23.9 & 23.0 & 23.5 \\
$2007-2008$ & 22.1 & 20.9 & 20.9 & 21.3 & 22.1 & 23.3 & 24.5 & 25.4 & 23.2 & 22.7 & 23.5 & 23.7 & 22.8
\end{tabular}




\section{Revista de CIENCIAS AMBIENTALES

\begin{tabular}{ccccccccccccccc}
\hline Fecha & Oct & Nov & Dic & Ene & Feb & Mar & Abr & May & Jun & Jul & Ago & Sep & Prom/año \\
\hline $2008-2009$ & 22.3 & 20.6 & 21.5 & 21.5 & 21.5 & 22.9 & 25.6 & 24.1 & 23.6 & 23.6 & 24.3 & 24.7 & 23.0 \\
$2009-2010$ & 23.4 & 22.2 & 22.5 & 21.3 & 23.4 & 24.8 & 24.7 & 24.3 & 23.9 & 23.6 & 23.3 & 23.0 & 23.4 \\
$2010-2011$ & 21.6 & 21.0 & 20.0 & 21.9 & 23.4 & 23.6 & 24.6 & 24.4 & 23.7 & 23.3 & 23.5 & 23.7 & 22.9 \\
$2011-2012$ & 21.5 & 21.7 & 20.9 & 21.4 & 22.3 & 24.4 & 23.6 & 23.6 & 23.5 & 23.4 & 23.1 & 23.1 & 22.7 \\
$2012-2013$ & 22.6 & 20.6 & 21.2 & 21.1 & 22.7 & 22.6 & 24.1 & 24.1 & 23.1 & 23.1 & 22.8 & 23.0 & 22.6 \\
$2013-2014$ & 23.1 & 21.8 & 22.2 & 21.4 & 21.5 & 23.8 & 25.1 & 23.3 & 23.4 & 23.8 & 24.4 & 23.3 & 23.1 \\
$2014-2015$ & 22.4 & 21.3 & 20.7 & 21.2 & 24.3 & 23.4 & 24.7 & 24.6 & 23.5 & 23.3 & 23.5 & 23.3 & 23.0 \\
$2015-2016$ & 23.6 & 22.7 & 23.0 & 21.8 & 21.4 & 24.2 & 25.9 & 25.6 & 23.4 & 23.6 & 23.9 & 23.6 & 23.6 \\
\hline Promedio & $\mathbf{2 2 . 7}$ & $\mathbf{2 1 . 6}$ & $\mathbf{2 1 . 4}$ & $\mathbf{2 1 . 3}$ & $\mathbf{2 2 . 5}$ & $\mathbf{2 3 . 8}$ & $\mathbf{2 4 . 8}$ & $\mathbf{2 4 . 6}$ & $\mathbf{2 3 . 6}$ & $\mathbf{2 3 . 4}$ & $\mathbf{2 3 . 7}$ & $\mathbf{2 3 . 4}$ & $\mathbf{2 3 . 1}$ \\
\hline
\end{tabular}

Apéndice 7. Consolidado mensual periodo 2001 al 2016, estación \#2, “Quiebramontes”. Appendix7. Monthly consolidated to 2001 to 2016, station \#2, "Quiebramontes"

\begin{tabular}{cccccccccccccc}
\hline Fecha & Oct & Nov & Dic & Ene & Feb & Mar & Abr & May & Jun & Jul & Ago & Sep & Prom/año \\
\hline $2000-2001$ & 20.6 & 21.1 & 19.5 & 19.2 & 20.1 & 21.2 & 23.8 & 22.9 & 22.7 & 22.0 & 23.0 & 21.8 & 21.5 \\
$2001-2002$ & 21.4 & 20.5 & 21.3 & 19.7 & 20.7 & 23.1 & 23.7 & 22.7 & 22.3 & 21.8 & 21.9 & 22.2 & 21.8 \\
$2002-2003$ & 21.3 & 19.6 & 19.8 & 18.7 & 20.7 & 23.8 & 23.2 & 24.5 & 23.0 & 23.0 & 22.7 & 23.0 & 21.9 \\
$2003-2004$ & 22.5 & 21.5 & 20.0 & 21.2 & 20.7 & 22.7 & 23.6 & 23.6 & 22.9 & 23.0 & 23.2 & 23.6 & 22.4 \\
$2004-2005$ & 23.3 & 21.7 & 21.0 & 20.9 & 20.7 & 26.4 & 25.3 & 24.0 & 23.6 & 23.3 & 23.7 & 23.2 & 23.1 \\
$2005-2006$ & 22.0 & 20.4 & 21.8 & 21.1 & 20.7 & 23.5 & 24.6 & 24.5 & 22.9 & 23.3 & 23.8 & 23.8 & 22.7 \\
$2006-2007$ & 23.7 & 21.0 & 21.4 & 21.6 & 20.7 & 23.9 & 23.9 & 25.4 & 23.9 & 23.4 & 23.7 & 23.4 & 23.0 \\
$2007-2008$ & 22.7 & 20.7 & 21.0 & 21.8 & 20.7 & 23.5 & 24.4 & 25.1 & 23.3 & 22.8 & 23.3 & 23.5 & 22.7 \\
$2008-2009$ & 22.4 & 20.9 & 21.5 & 21.3 & 21.6 & 23.0 & 26.6 & 24.4 & 23.9 & 23.9 & 24.3 & 24.5 & 23.2 \\
$2009-2010$ & 24.2 & 22.7 & 22.7 & 21.5 & 20.6 & 25.4 & 25.1 & 24.4 & 24.4 & 24.4 & 23.8 & 24.7 & 23.7 \\
$2010-2011$ & 24.4 & 23.8 & 22.6 & 23.1 & 20.6 & 24.4 & 25.3 & 25.1 & 24.9 & 24.9 & 25.1 & 25.2 & 24.1 \\
$2011-2012$ & 24.5 & 23.4 & 22.8 & 23.3 & 20.6 & 24.4 & 24.9 & 24.4 & 24.8 & 24.2 & 24.6 & 24.5 & 23.9 \\
$2012-2013$ & 23.9 & 22.8 & 22.9 & 23.6 & 20.6 & 24.8 & 25.6 & 24.7 & 23.9 & 24.1 & 24.2 & 23.9 & 23.8 \\
$2013-2014$ & 24.5 & 23.8 & 23.5 & 22.8 & 20.6 & 25.0 & 25.8 & 25.3 & 24.2 & 25.1 & 25.5 & 24.9 & 24.3 \\
$2014-2015$ & 24.7 & 24.1 & 23.6 & 23.1 & 20.6 & 23.3 & 26.7 & 26.5 & 25.2 & 25.4 & 26.4 & 25.8 & 24.6 \\
$2015-2016$ & 25.8 & 25.2 & 25.0 & 24.3 & 20.6 & 25.9 & 27.7 & 27.7 & 25.4 & 25.5 & 26.4 & 25.7 & 25.4 \\
\hline Promedio & 23.2 & $\mathbf{2 2 . 1}$ & $\mathbf{2 1 . 9}$ & $\mathbf{2 1 . 7}$ & $\mathbf{2 0 . 7}$ & $\mathbf{2 4 . 0}$ & $\mathbf{2 5 . 0}$ & $\mathbf{2 4 . 7}$ & $\mathbf{2 3 . 8}$ & $\mathbf{2 3 . 6}$ & $\mathbf{2 4 . 1}$ & $\mathbf{2 4 . 0}$ & $\mathbf{2 3 . 2}$ \\
\hline & & & & & & & & & & & &
\end{tabular}




\section{Revista de CIENCIAS AMBIENTALES Tropical Journal of Environmental Sciences}

Revista de Ciencias Ambientales (Trop J Environ Sci) e-ISSN: 2215-3896 (Enero-Junio, 2021) . Vol 55(1): 118-142 DOI: https://doi.org/10.15359/rca.55-1.6 Open Access: www.revistas.una.ac.cr/ambientales e-mail: revista.ambientales@una.ac.cr Rodríguez-Cruz G.

Apéndice 8. Consolidado mensual periodo 2004 al 2012, estación \#3, "El Escarbadero". Appendix 8. Monthly consolidated to 2004 to 2012, station \#3, "El Escarbadero"

\begin{tabular}{cccccccccccccc}
\hline Fecha & Oct & Nov & Dic & Ene & Feb & Mar & Abr & May & Jun & Jul & Ago & $\begin{array}{c}\text { Sep } \\
\text { Prom/ } \\
\text { año }\end{array}$ \\
\hline $2003-2004$ & 19.6 & 18.6 & 17.1 & 18.3 & 17.8 & 19.8 & 20.7 & 20.7 & 20.0 & 20.1 & 20.3 & 20.7 & 19.5 \\
$2004-2005$ & 20.4 & 18.8 & 18.1 & 18.0 & 17.8 & 23.5 & 22.4 & 21.1 & 20.7 & 20.4 & 20.8 & 20.3 & 20.2 \\
$2005-2006$ & 19.1 & 17.5 & 18.9 & 18.2 & 17.8 & 20.6 & 21.7 & 21.6 & 20.0 & 20.4 & 20.9 & 20.9 & 19.8 \\
$2006-2007$ & 20.8 & 18.1 & 18.5 & 18.7 & 17.8 & 21.0 & 21.0 & 22.5 & 21.0 & 20.5 & 20.8 & 20.5 & 20.1 \\
$2007-2008$ & 19.8 & 17.8 & 18.1 & 18.9 & 17.8 & 20.6 & 21.5 & 22.2 & 20.4 & 19.9 & 20.4 & 20.6 & 19.8 \\
$2008-2009$ & 19.5 & 18.0 & 18.6 & 18.4 & 18.7 & 20.1 & 23.7 & 21.5 & 21.0 & 21.0 & 21.4 & 21.6 & 20.3 \\
$2009-2010$ & 21.3 & 19.8 & 19.8 & 18.6 & 17.7 & 22.5 & 22.2 & 21.5 & 21.5 & 21.5 & 20.9 & 21.8 & 20.8 \\
$2010-2011$ & 21.5 & 20.9 & 19.7 & 20.2 & 17.7 & 21.5 & 22.4 & 22.2 & 22.0 & 22.0 & 22.2 & 22.3 & 21.2 \\
$2011-2012$ & 21.6 & 20.5 & 19.9 & 20.4 & 17.7 & 21.5 & 22.0 & 21.5 & 21.9 & 21.3 & 21.7 & 21.6 & 21.0 \\
\hline Promedio & $\mathbf{2 0 . 4}$ & $\mathbf{1 8 . 9}$ & $\mathbf{1 8 . 7}$ & $\mathbf{1 8 . 9}$ & $\mathbf{1 7 . 9}$ & $\mathbf{2 1 . 2}$ & $\mathbf{2 2 . 0}$ & $\mathbf{2 1 . 6}$ & $\mathbf{2 0 . 9}$ & $\mathbf{2 0 . 8}$ & $\mathbf{2 1 . 0}$ & $\mathbf{2 1 . 1}$ & $\mathbf{2 0 . 3}$ \\
\hline
\end{tabular}

Apéndice 9. Consolidado mensual periodo 2004 al 2016, estación \#4, "Rincón de Dolores".

Appendix 9. Monthly consolidated to 2004 to 2016, station \#4, "Rincón de Dolores"

\begin{tabular}{cccccccccccccc}
\hline Fecha & Oct & Nov & Dic & Ene & Feb & Mar & Abr & May & Jun & Jul & Ago & Sep & Prom/año \\
\hline $2003-2004$ & 19.6 & 18.6 & 17.1 & 18.3 & 17.8 & 19.8 & 20.7 & 20.7 & 20.0 & 20.1 & 20.3 & 20.7 & 19.5 \\
$2004-2005$ & 20.4 & 18.8 & 18.1 & 18.0 & 17.8 & 23.5 & 22.4 & 21.1 & 20.7 & 20.4 & 20.8 & 20.3 & 20.2 \\
$2005-2006$ & 19.1 & 17.5 & 18.9 & 18.2 & 17.8 & 20.6 & 21.7 & 21.6 & 20.0 & 20.4 & 20.9 & 20.9 & 19.8 \\
$2006-2007$ & 20.8 & 18.1 & 18.5 & 18.7 & 17.8 & 21.0 & 21.0 & 22.5 & 21.0 & 20.5 & 20.8 & 20.5 & 20.1 \\
$2007-2008$ & 19.8 & 17.8 & 18.1 & 18.9 & 17.8 & 20.6 & 21.5 & 22.2 & 20.4 & 19.9 & 20.4 & 20.6 & 19.8 \\
$2008-2009$ & 19.5 & 18.0 & 18.6 & 18.4 & 18.7 & 20.1 & 23.7 & 21.5 & 21.0 & 21.0 & 21.4 & 21.6 & 20.3 \\
$2009-2010$ & 21.3 & 19.8 & 19.8 & 18.6 & 17.7 & 22.5 & 22.2 & 21.5 & 21.5 & 21.5 & 20.9 & 21.8 & 20.8 \\
$2010-2011$ & 21.5 & 20.9 & 19.7 & 20.2 & 17.7 & 21.5 & 22.4 & 22.2 & 22.0 & 22.0 & 22.2 & 22.3 & 21.2 \\
$2011-2012$ & 21.6 & 20.5 & 19.9 & 20.4 & 17.7 & 21.5 & 22.0 & 21.5 & 21.9 & 21.3 & 21.7 & 21.6 & 21.0 \\
$2012-2013$ & 21.0 & 19.9 & 20.0 & 20.7 & 17.7 & 21.9 & 22.7 & 21.8 & 21.0 & 21.2 & 21.3 & 21.0 & 20.9 \\
$2013-2014$ & 21.6 & 20.9 & 20.6 & 19.9 & 17.7 & 22.1 & 22.9 & 22.4 & 21.3 & 22.2 & 22.6 & 22.0 & 21.4 \\
$2014-2015$ & 21.8 & 21.2 & 20.7 & 20.2 & 17.7 & 20.4 & 23.8 & 23.6 & 22.3 & 22.5 & 23.5 & 22.9 & 21.7 \\
$2015-2016$ & 22.9 & 22.3 & 22.0 & 21.4 & 17.7 & 23.0 & 24.8 & 24.8 & 22.5 & 22,6 & 23.5 & 22.8 & 22.5 \\
\hline Promedio & $\mathbf{2 0 . 8}$ & $\mathbf{1 9 . 6}$ & $\mathbf{1 9 . 4}$ & $\mathbf{1 9 . 4}$ & $\mathbf{1 7 . 8}$ & $\mathbf{2 1 . 4}$ & $\mathbf{2 2 . 4}$ & $\mathbf{2 2 . 1}$ & $\mathbf{2 1 . 2}$ & $\mathbf{2 1 . 2}$ & $\mathbf{2 1 . 6}$ & $\mathbf{2 1 . 5}$ & $\mathbf{2 0 . 7}$ \\
\hline
\end{tabular}




\section{Revista de CIENCIAS AMBIENTALES Tropical Journal of Environmental Sciences}

Revista de Ciencias Ambientales (Trop J Environ Sci) e-ISSN: 2215-3896

(Enero-Junio, 2021) . Vol 55(1): 118-142 DOI: https://doi.org/10.15359/rca.55-1.6 Open Access: www.revistas.una.ac.cr/ambientales e-mail: revista.ambientales@una.ac.cr Rodríguez-Cruz G.

Apéndice 10. Consolidado mensual periodo 2001 al 2016, estación \#5, "San Matías". Appendix 10. Monthly consolidated to 2001 to 2016, station \#5, "San Matías"

\begin{tabular}{cccccccccccccc}
\hline Fecha & Oct & Nov & Dic & Ene & Feb & Mar & Abr & May & Jun & Jul & Ago & Sep & Prom/año \\
\hline $2000-2001$ & 19.2 & 19.7 & 18.1 & 17.8 & 18.7 & 19.8 & 22.4 & 21.5 & 21.3 & 20,6 & 21.6 & 20.4 & 20.1 \\
$2001-2002$ & 20.0 & 19.1 & 19.9 & 18.3 & 19.3 & 21.7 & 22.3 & 21.3 & 20.9 & 20,4 & 20.5 & 20.8 & 20.4 \\
$2002-2003$ & 19.9 & 18.2 & 18.4 & 17.3 & 19.3 & 22.4 & 21.8 & 23.1 & 21.6 & 21,6 & 21.3 & 21.6 & 20.5 \\
$2003-2004$ & 21.1 & 20.1 & 18.6 & 19.8 & 19.3 & 21.3 & 22.2 & 22.2 & 21.5 & 21,6 & 21.8 & 22.2 & 21.0 \\
$2004-2005$ & 21.9 & 20.3 & 19.6 & 19.5 & 19.3 & 25.0 & 23.9 & 22.6 & 22.2 & 21,9 & 22.3 & 21.8 & 21.7 \\
$2005-2006$ & 20.6 & 19.0 & 20.4 & 19.7 & 19.3 & 22.1 & 23.2 & 23.1 & 21.5 & 21,9 & 22.4 & 22.4 & 21.3 \\
$2006-2007$ & 22.3 & 19.6 & 20.0 & 20.2 & 19.3 & 22.5 & 22.5 & 24.0 & 22.5 & 22,0 & 22.3 & 22.0 & 21.6 \\
$2007-2008$ & 21.3 & 19.3 & 19.6 & 20.4 & 19.3 & 22.1 & 23.0 & 23.7 & 21.9 & 21,4 & 21.9 & 22.1 & 21.3 \\
$2008-2009$ & 21.0 & 19.5 & 20.1 & 19.9 & 20.2 & 21.6 & 25.2 & 23.0 & 22.5 & 22,5 & 22.9 & 23.1 & 21.8 \\
$2009-2010$ & 22.8 & 21.3 & 21.3 & 20.1 & 19.2 & 24.0 & 23.7 & 23.0 & 23.0 & 23,0 & 22.4 & 23.3 & 22.3 \\
$2010-2011$ & 23.0 & 22.4 & 21.2 & 21.7 & 19.2 & 23.0 & 23.9 & 23.7 & 23.5 & 23,5 & 23.7 & 23.8 & 22.7 \\
$2011-2012$ & 23.1 & 22.0 & 21.4 & 21.9 & 19.2 & 23.0 & 23.5 & 23.0 & 23.4 & 22,8 & 23.2 & 23.1 & 22.5 \\
$2012-2013$ & 22.5 & 21.4 & 21.5 & 22.2 & 19.2 & 23.4 & 24.2 & 23.3 & 22.5 & 22,7 & 22.8 & 22.5 & 22.4 \\
$2013-2014$ & 23.1 & 22.4 & 22.1 & 21.4 & 19.2 & 23.6 & 24.4 & 23.9 & 22.8 & 23,7 & 24.1 & 23.5 & 22.9 \\
$2014-2015$ & 23.3 & 22.7 & 22.2 & 21.7 & 19.2 & 21.9 & 25.3 & 25.1 & 23.8 & 24,0 & 25.0 & 24.4 & 23.2 \\
$2015-2016$ & 23.9 & 23.3 & 23.0 & 22.4 & 18.7 & 24.0 & 25.8 & 25.8 & 23.5 & 23,6 & 24.5 & 23.8 & 23.5 \\
\hline Promedio & $\mathbf{2 1 . 8}$ & $\mathbf{2 0 . 6}$ & $\mathbf{2 0 . 5}$ & $\mathbf{2 0 . 3}$ & $\mathbf{1 9 . 2}$ & $\mathbf{2 2 . 6}$ & $\mathbf{2 3 . 6}$ & $\mathbf{2 3 . 3}$ & $\mathbf{2 2 . 4}$ & $\mathbf{2 2 , 3}$ & $\mathbf{2 2 . 7}$ & $\mathbf{2 2 . 6}$ & $\mathbf{2 1 . 8}$ \\
\hline & & & & & & & & & & & &
\end{tabular}

